\title{
High expression of Ran binding protein 1 predicts poor outcomes in hepatocellular carcinoma patients: a Cancer Genome Atlas database analysis
}

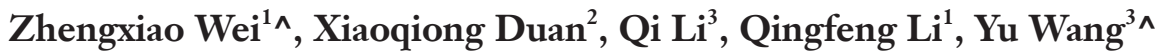 \\ ${ }^{1}$ Department of Clinical Laboratory, Public Health Clinical Center of Chengdu, Chengdu, China; ${ }^{2}$ Institute of Blood Transfusion, Chinese \\ Academy of Medical Sciences and Peking Union Medical College, Chengdu, China; ${ }^{3}$ College of Bioinformatics, Chongqing University of Posts and \\ Telecommunications, Chongqing Key Laboratory of Big Data for Bio-intelligence, Chongqing, China \\ Contributions: (I) Conception and design: Z Wei, Y Wang; (II) Administrative support: None; (III) Provision of study materials or patients: None; \\ (IV) Collection and assembly of data: X Duan, Q Li; (V) Data analysis and interpretation: Q Li, Y Wang, X Duan, Q Li; (VI) Manuscript writing: All \\ authors; (VII) Final approval of manuscript: All authors. \\ Correspondence to: Professor Yu Wang. College of Bioinformatics, Chongqing University of Posts and Telecommunications, Chongqing Key \\ Laboratory of Big Data for Bio-intelligence, 2 Chongwen Road, Nan’an District, Chongqing 400065, China. Email: wangyu@cqupt.edu.cn.
}

Background: Ran-specific binding protein 1 (RANBP1) is involved in the regulation of the cell cycle, while its role in hepatocellular carcinoma (HCC) is unknown. Therefore, we aimed to demonstrate the association of RANBP1 with clinicopathologic features and potential biological functions in HCC based on The Cancer Genome Atlas (TCGA) data.

Methods: We assessed $R A N B P 1$ expression and its correlation with clinicopathologic features and evaluated the prognostic value of $R A N B P 1$ with Kaplan-Meier survival analysis and the MethSurv database. Univariate and multivariate Cox regression analyses were conducted to elucidate the factors responsible for prognosis. The identification of a co-expression network and the analysis of related biological events with RANBP1 in HCC were assessed using LinkedOmics. Moreover, gene set enrichment analysis (GSEA) was employed to annotate the biological function of RANBP1. We also explored the correlation between RANBP1 and tumor immune infiltrates using a single sample GSEA (ssGSEA).

Results: The expression of RANBP1 was found significantly elevated in HCC and linked to advanced T stage and histopathological grade. Up-regulated $R A N B P 1$ expression was linked to poor prognosis. High DNA methylation levels of $R A N B P 1$ were significantly linked to very poor overall survival (OS). Coexpression network analysis revealed that $R A N B P 1$ was involved in ribosome, spliceosome, deoxyribonucleic acid (DNA) replication, ribonucleic acid (RNA) transport, and cell cycle. GSEA showed enrichment of G2M-checkpoint, Wingless and Int-1 (Wnt) cell signaling, and DNA repair in the RANBP1 high-expression phenotype. By using ssGSEA analysis, the increased $R A N B P 1$ expression was positively linked to the immune infiltration level of T helper cell type-1 (Th1) and negatively linked to the immune infiltration levels of T helper cell type-17 (Th17).

Conclusions: Findings suggest that RANBP1 may play a pivotal role in HCC prognosis and can potentially serve as a candidate biosignature and as a therapeutic target for HCC.

Keywords: Hepatocellular carcinoma (HCC); prognosis; immune infiltration; Ran-specific binding protein 1 (RANBP1); The Cancer Genome Atlas database (TCGA database)

Submitted Jun 23, 2021. Accepted for publication Oct 14, 2021.

doi: 10.21037/jgo-21-541

View this article at: https://dx.doi.org/10.21037/jgo-21-541

^ ORCID: Zhengxiao Wei, 0000-0001-5065-7623; Yu Wang, 0000-0002-8955-7029. 


\section{Introduction}

Hepatocellular carcinoma (HCC) is among the most frequent malignant tumors and has a very poor prognosis. It is the fifth and ninth most frequent cancer in males and females respectively, and the second principal cause of cancer death worldwide $(1,2)$. At present, surgical resection, transcatheter arterial chemoembolization, transcatheter arterial chemoinfusion, radiofrequency ablation, and molecular targeted drug therapy are standard treatment methods for HCC, which greatly improve the survival of HCC patients (3). However, even after curative treatment, the recurrence rate of HCC in patients is still more than $70 \%$ at 5 years (4). Only $46 \%$ of patients are diagnosed at an early stage (5), and many patients at intermediate and advanced stages cannot be cured. Although an increasing number of studies have assessed prognostic biomarkers (6-8), it remains crucial to investigate more thoroughly potential biomarkers that can reliably detect HCC to allow identification of HCC patients with poor prognoses. In recent study, the immune and scores of 371 cases were calculated for quantitative analysis of immune and stromal cell infiltration in the tumour microenvironment of hepatocellular carcinoma (HCC). The weighted gene co-expression network analysis and proteinprotein interaction network were analyzed to identify immune microenvironment-related genes (9). Therefore, screening HCC prognostic immune-related genes through bioinformatics methods and constructing a prognostic risk scoring model based on immune-related gene signatures can provide a basis for the prognosis evaluation of HCC patients and the clinical decision-making of individualized treatment (10).

Previous studies $(11,12)$ have demonstrated that Ran-specific binding protein 1 (RANBP1) plays a key role in Ras-related nuclear protein (RAN) dependent nucleocytoplasmic transport. Guanosine-5'-triphosphate (GTP) enzyme activator activity and guanosine 5 '-diphosphate (GDP)-dissociation inhibitor activity are closely related to RANBP1. As the nuclear transport effector, RANBP1 combined with RAN-GTP/GDP and Ran GTPase-activating protein 1 (RANGAP1) form the Ran network (13), which has been implicated in the control of chromosome segregation (14) and proliferation (12) and mitotic stability (15). Recently, literature reported that RANBP1 was up-regulated and could serve as a promising therapeutic target in bladder cancer (16). Recent studies have found that RANBP1 might be up-regulated by the over-expression of serum and glucocorticoid-regulated kinase 1 (SGK1) and promoted tumor cell proliferation (13). As far as the authors are aware, RANBP1 expression level and its prognostic value in HCC remain unclear. Therefore, the present study focused on RANBP1 and investigated its potential role in HCC. In this study, the potential role of RANBP1 in HCC was studied through the analysis of the cancer genome atlas database, which provided new insights into the pathogenesis of HCC, and may serve as a new candidate biomarker and therapeutic target for HCC. We present the following article in accordance with the REMARK reporting checklist (available at https://dx.doi. org/10.21037/jgo-21-541).

\section{Methods}

\section{Ribonucleic acid (RNA)-sequence data and clinic information of HCC from The Cancer Genome Atlas (TCGA) and UCSC Xena}

In this study, Ran-sequence data for 371 patients with HCC and 50 cases of neighboring healthy tissues and matching patients' clinical information were collected from the Liver Hepatocellular Carcinoma (LIHC) project of TCGA (https://portal.gdc.cancer.gov/). The RNA-sequence data combined TCGA and genotype-tissue expression (GTEx) healthy specimens through toil process (17) and were abstracted from the UCSC Xena web resource (https:// xenabrowser.net/datapages/). We discarded the data without clinical information or gene expression values. Clinicopathological parameters included age, gender, pathologic stage, tumor/node/metastases (T/N/M) stage, residual tumor, fibrosis Ishak score, vascular invasion, Child-Pugh grade, and race (these data are summarized in Table 1). Comparisons of the gene expression profiles of the tumors with those of neighboring healthy tissues of HCC were carried out. We transformed level 3 HTSeq-FPKM data to transcripts per million reads (TPM) for further analyses. This study met TCGA publication guidelines. The study was conducted in accordance with Declaration of Helsinki (as revised in 2013).

\section{Validation of differential expression in the Integrative Molecular Database for Hepatocellular Carcinoma (HCCDB) and Human Protein Atlas (HPA) database}

The HCCDB database (http://lifeome.net/database/hccdb/) is an online and user-friendly website that can provide 
Table 1 Clinicopathological characteristics of high-/low-RANBP1 expression group in HCC

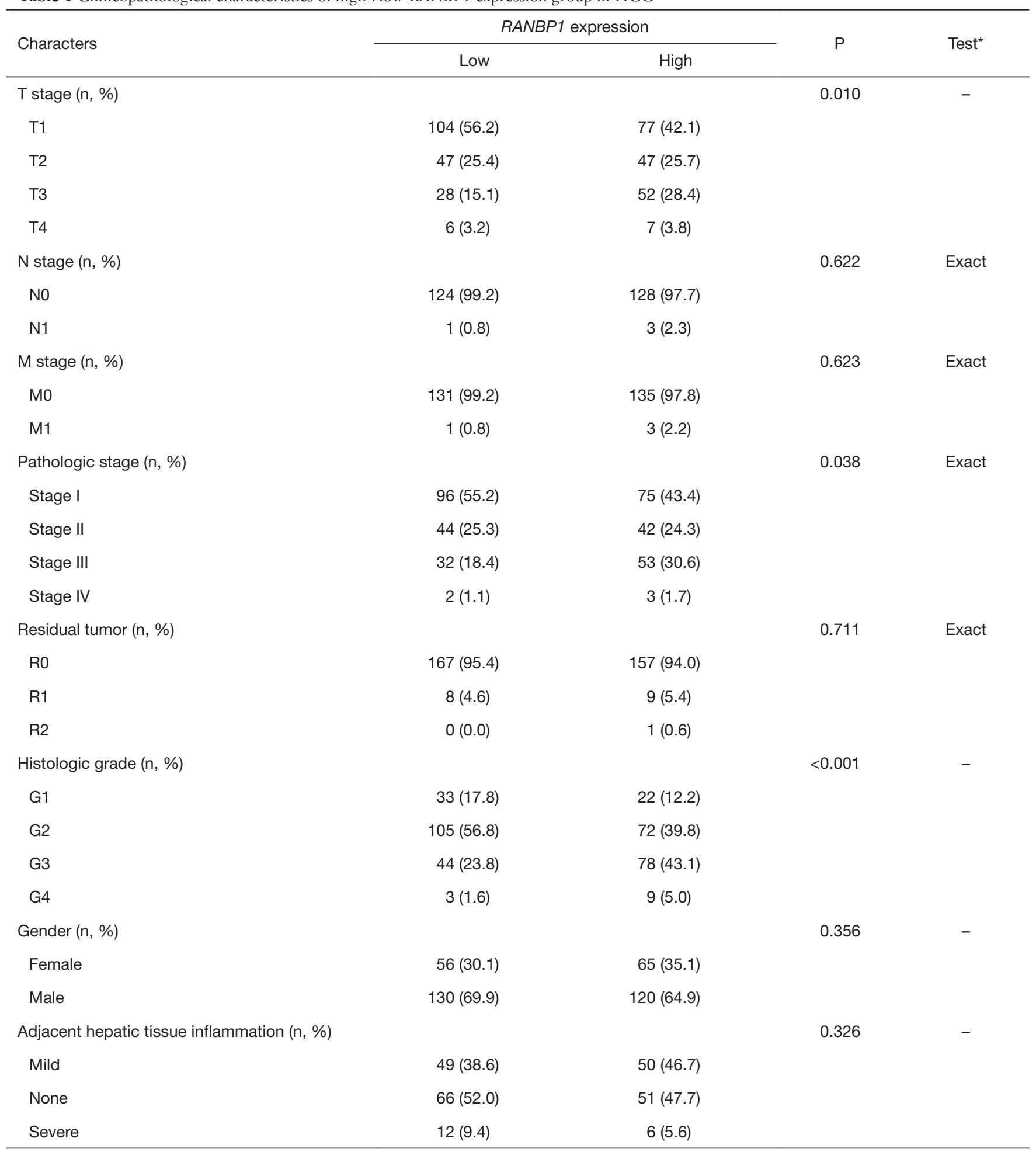

Table 1 (continued) 
Table 1 (continued)

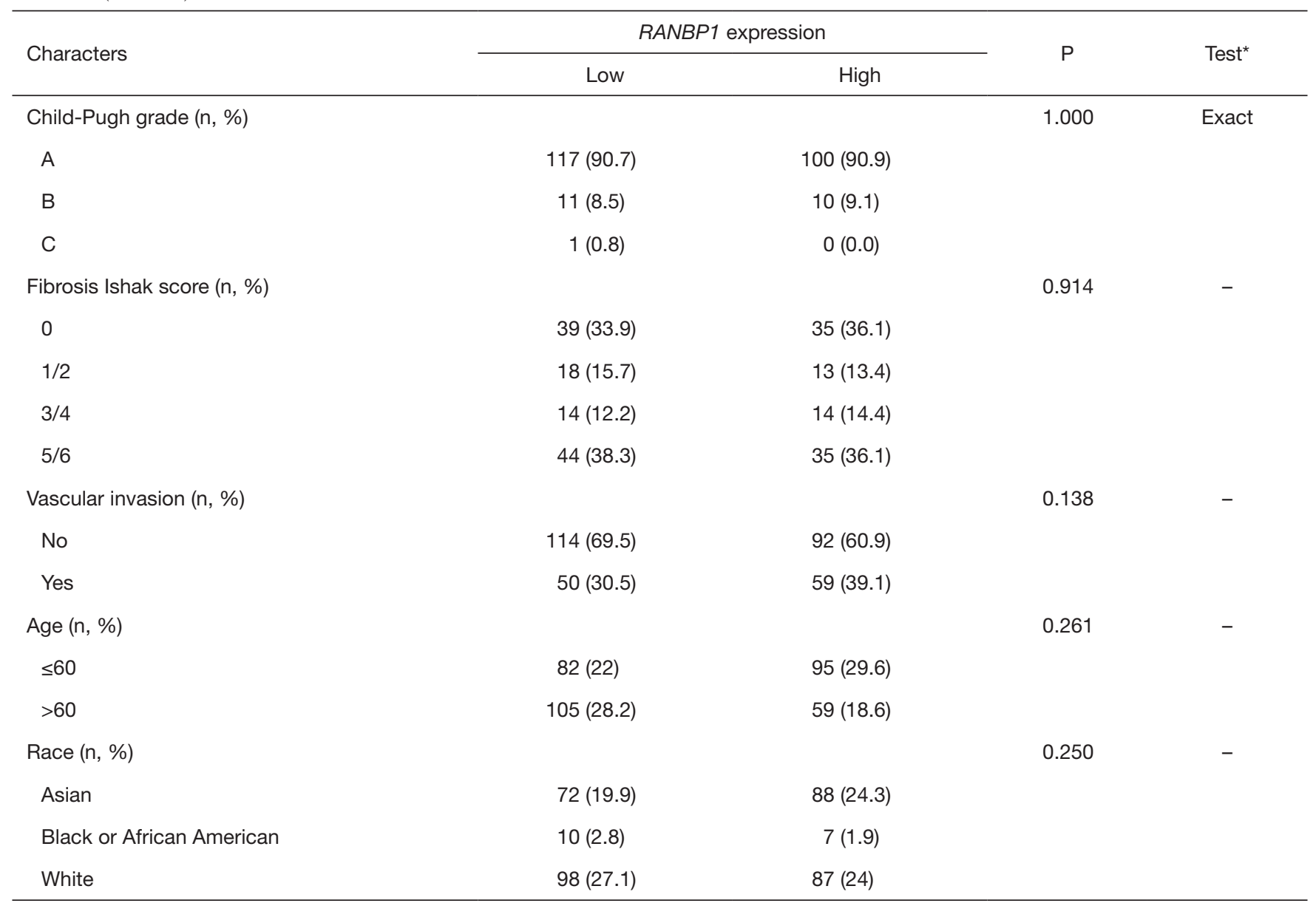

*, Chi-square test is used by default for categorical variables, and 'exact' means that the statistical method is Fisher's exact test. RANBP1,

Ran-specific binding protein 1; HCC, hepatocellular carcinoma.

the expression patterns and the annotation of transcripts of HCC genes (18); it was further utilized to assess the expressing level and co-expressed network of RANBP1 in HCC. In parallel, HPA (https://www.proteinatlas.org/) is a public database that describes all the human proteins in cell lines, tissues, and organs using immunohistochemistry (19). In our study, HPA was utilized to evaluate the protein expression of RANBP1 among HCC patients' tissues and normal human tissues.

\section{Statistical analysis to explore relationships between RANBP1 and clinicopathological factors}

The differences in the RANBP1 expressions between healthy tissues and tumors in HCC cohorts are shown in box figures, which were prepared using ggplot2 in $\mathrm{R}$
Version 3.6.2 (The R Foundation for Statistical Computing, Vienna, Austria). Kruskal-Wallis tests, Spearman's rank correlations and Wilcoxon signed-rank tests were used to explore the relationships between the clinicopathological features and RANBP1 expressions. Receiver operating characteristic (ROC) analysis was performed by $\mathrm{R}$ package survival ROC Version 1.0.3 (The R Foundation for Statistical Computing, Vienna, Austria). Moreover, KaplanMeier curves were employed to measure overall survival (OS), progression-free interval (PFI), and the diseasespecific survival (DSS) difference between the high and low RANBP1 expression groups in the HCC cohorts of TCGA by using the survminer $\mathrm{R}$ package (The $\mathrm{R}$ Foundation for Statistical Computing, Vienna, Austria). The differences between the survival curves were examined by the log-rank test. The prognostic data of PFI and DSS were obtained 
from the article published by Liu et al. (20). Univariate and multivariate Cox regressions were employed to evaluate the expression of RANBP1 and the survival rate of patients with given clinical factors (age, sex, grade, clinical stage, T/ N/M stage, etc.) (21). During all the analyses in this study, a statistically significant difference was defined as a $\mathrm{P}$ value less than 0.05 .

\section{MethSurv database analysis}

MethSurv (https://biit.cs.ut.ee/methsurv/) is a web tool for visualization of methylation biomarkers and their survival analysis based on the patterns of CpG methylation (22). We employed this web tool to analyze the DNA methylation sites of RANBP1 in TCGA database and to perform survival analysis of all the methylation sites.

\section{Co-expression network and bub gene selection}

To further identify the co-expression network, the LinkedOmics database (21) (http://www.linkedomics.org/ admin.php) was requisitioned; it allows for analysis of multi-omics data within and across 32 cancer types (23). The filter conditions were as follows: cancer cohort: TCGA_LIHC; search dataset: TCGA_LIHC (RNAseq); search dataset attribute: RANBP1; search target dataset: TCGA_UCEC (RNAseq); and search statistical method: Pearson correlation coefficient test. The top 100 positive co-expression genes were mapped onto the STRING website (https://string-db.org/) (24) to enroll in the proteinprotein interaction (PPI) network, and the PPI network was visualized in Cytoscape (Version 3.7.1) software. Subsequently, hub genes were selected using the Molecular Complex Detection (MCODE) plug-in.

\section{Gene set enrichment analysis (GSEA)}

GSEA (25) was performed using the gseGO and gseKEGG function of the clusterProfiler package Version 3.14.3 (Bioconductor, Roswell Park Comprehensive Cancer Center, Buffalo, NY, USA) (26), respectively, to assess the underlying function and pathway difference between highand low-RANBP1 expression sections. The adjusted $\mathrm{P}$-value $(<0.05)$, false discovery rate (FDR) q-value $(<0.25)$, and normalized enrichment score $(|\mathrm{NES}|>1)$ were employed to sort the pathway enriched in each phenotype. Based on the default-weighted enrichment statistical method, the gene set permutations procedure was repeated 1,000 times for each assessment.

\section{Correlation between RANBP1 expression and immune infiltration}

The infiltration analysis of the immune cell was assessed via single-sample GSEA (ssGSEA) using R package GSVA Version 3.6.3 (The R Foundation for Statistical Computing, Vienna, Austria) (27), in which the enrichment score was displayed in a sample as per the expression levels of immune cell-distinct marker genes. Marker genes for 24 immune cells in tissues were acquired from the Bindea et al. (28) study. The analysis of ssGSEA was performed based on the GenePattern environment (Broad Institute, Cambridge, MA, USA) (29). To explore the relationship of $R A N B P 1$ with the infiltration levels of immune cells and the association of invasion of immune cells with the different expression groups of RANBP1, the Wilcoxon rank sum test and Spearman's correlation test were used.

\section{Statistical analysis}

We assessed RANBP1 expression and its correlation with clinicopathologic features and evaluated the prognostic value of RANBP1 with Kaplan-Meier survival analysis and the MethSurv database. Univariate and multivariate Cox regression analyses were conducted to elucidate the factors responsible for prognosis. The identification of a co-expression network and the analysis of related biological events with RANBP1 in HCC were assessed using LinkedOmics. Moreover, gene set enrichment analysis (GSEA) was employed to annotate the biological function of $R A N B P 1$. We also explored the correlation between $R A N B P 1$ and tumor immune infiltrates using a single sample GSEA (ssGSEA).

\section{Results}

\section{Expression of RANBP1 in tumors and neighboring bealthy} tissues of HCC

We first compared the expression of RANBP1 in tumors and neighboring healthy tissues of HCC patients through analyzing data abstracted from TCGA. As indicated in Figure 1A, the expression of RANBP1 in the tumors was significantly up-regulated compared with that in neighboring healthy tissues $(\mathrm{P}<0.001)$. Given the data 
imbalance in the tumors (371 cases) and neighboring healthy tissues (50 cases), we also measured the expression profiles of $R A N B P 1$ in the neighboring healthy tissues and their paired tumors, and the RANBP1 expression in the tumors was still significantly higher in contrast to that in neighboring healthy tissues (Figure $1 B, \mathrm{P}<0.001$ ).

To provide a more comprehensive evaluation of RANBP1 expression in tumors, we integrated the RNAsequence data in TPM format from TCGA and the GTEx cohort from UCSC Xena, using the UCSC Toil process to remove batch effects and to allow for sample merging (17). As shown in Figure 1C, the expression of RANBP1 in the tumors was still noticeably higher than that in healthy tissues $(\mathrm{P}<0.001)$.

To explore the potential application value of RANBP1 in the diagnosis of HCC, we employed a ROC curve. As indicated in Figure 1D, the area of under the curve (AUC) was 0.840 , showing that RANBP1 exhibited good classification accuracy and predictive power in patients with HCC.

We next validated the differential transcription levels of RANBP1 in the HCCDB database with multiple HCC clinical cohorts. Through the analysis of 7 out of $10 \mathrm{HCC}$ clinical cohorts, we found that messenger ribonucleic acid (mRNA) expression of RANBP1 in HCC tissues was significantly up-regulated compared with that in normal liver tissues (Figure S1A). Moreover, HPA was requisitioned used to assess the protein expression levels of RANBP1 in normal and cancer tissues. As shown in Figure $\mathrm{S} 1 \mathrm{~B}$, the $R A N B P 1$ protein level assessed using antibody HPA0659341 was overexpressed in HCC samples compared with that in normal liver samples (Figure S1B,S1C). In general, both mRNA and protein level for RANBP1 expression was significantly up-regulated in HCC.

We also compared $R A N B P 1$ expression between tumor and neighboring samples in 33 cancer types in TCGA (Figure $1 E$ ) and the UCSC Xena cohort (Figure $1 F$ ). It was observed that $R A N B P 1$ was also overexpressed in the following tumors, with a $|\log 2 \mathrm{FC}|$ cutoff $>1$, and a $\mathrm{P}$ value $<0.05$, including breast invasive carcinoma (BRCA), LIHC, bladder urothelial carcinoma (BLCA), cervical squamous cell carcinoma and endocervical adenocarcinoma (CESC), cholangiocarcinoma (CHOL), esophageal carcinoma (ESCA), colon adenocarcinoma (COAD), glioblastoma multiform (GBM), head and neck squamous cell carcinoma (HNSC), kidney renal clear cell carcinoma (KIRC), lung adenocarcinoma (LUAD), prostate adenocarcinoma
(PRAD), lung squamous cell carcinoma (LUSC), rectal adenocarcinoma (READ), sarcoma (SARC), stomach adenocarcinoma (STAD), uterine corpus endometrial carcinoma (UCEC), etc. These results revealed that $R A N B P 1$ might play an oncogenic role in HCC progression.

\section{Clinicopathological characteristics of high-/low-RANBP1 expression group in $\mathrm{HCC}$}

To evaluate whether the expression levels of $R A N B P 1$ are related to the progression of HCC, we explored the correlations between $R A N B P 1$ expression and the clinicopathological features in 371 individuals with HCC. For this assessment, patient specimens were clustered into two groups on the basis of the median expression level of $R A N B P 1$, i.e., the high-expression group and the lowexpression group for further study. The characteristics of HCC patients in TCGA including gender, race, T/N/M stage, histologic grade, pathologic stage, and vascular invasion were collected, as shown in Table 1. In general, we found the RANBP1 expression level was significantly correlated with $\mathrm{T}$-stage $(\mathrm{P}=0.01)$, pathologic stage $(\mathrm{P}=0.038)$, and histologic grade $(\mathrm{P}<0.001)$ using Fisher's exact test or chi-square test. No correlation was found between RANBP1 expression and the other clinicopathologic features (Table 1 and Figure $2 A-2 C$ ).

As shown in Figure $2 D-2 F$, using further sub-group analyses, the expression profiles of $R A N B P 1$ performed significantly differently in $T$ stage $(\mathrm{P}=0.01)$, pathologic stage $(\mathrm{P}=0.038)$, and histologic grade $(\mathrm{P}<0.001)$, and it showed higher $R A N B P 1$ expression levels linked to advanced $\mathrm{T}$ stage and histologic grade. Thus, RANBP1 expression was involved in speeding up the process of HCC.

\section{Survival outcomes and multivariate analyses}

Kaplan-Meier survival analysis was used to assess the relationship between $R A N B P 1$ expression levels and prognosis. As shown in Figure 3A-3C, the Kaplan-Meier survival analysis demonstrated that $\mathrm{HCC}$ with high RANBP1 expression was linked to very poor OS (HR: 1.95; 95\% CI: 1.36-2.78; $\mathrm{P}<0.001$ ), unfavorable PFI (HR: 1.58; 95\% CI: $1.17-2.12 ; \mathrm{P}=0.002$ ), and worse DSS (HR: 2.12; 95\% CI: $1.34-3.36 ; \mathrm{P}=0.001)$ compared with the low $R A N B P 1$ expression group. Furthermore, we analyzed the association between $R A N B P 1$ expression level and survival in the subgroup of patients with low histologic grades (G1 and G2). The results showed that the high RANBP1 

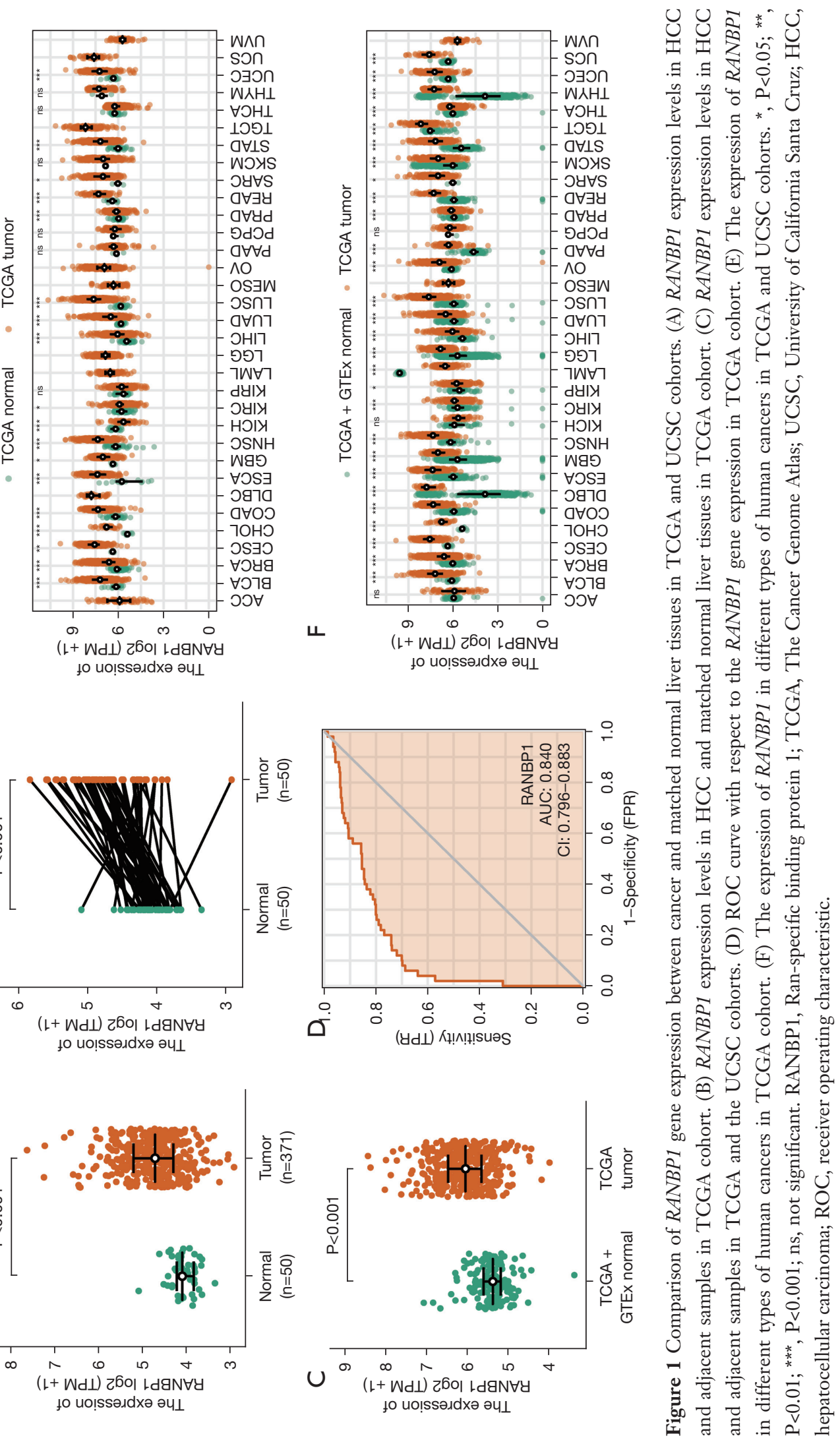

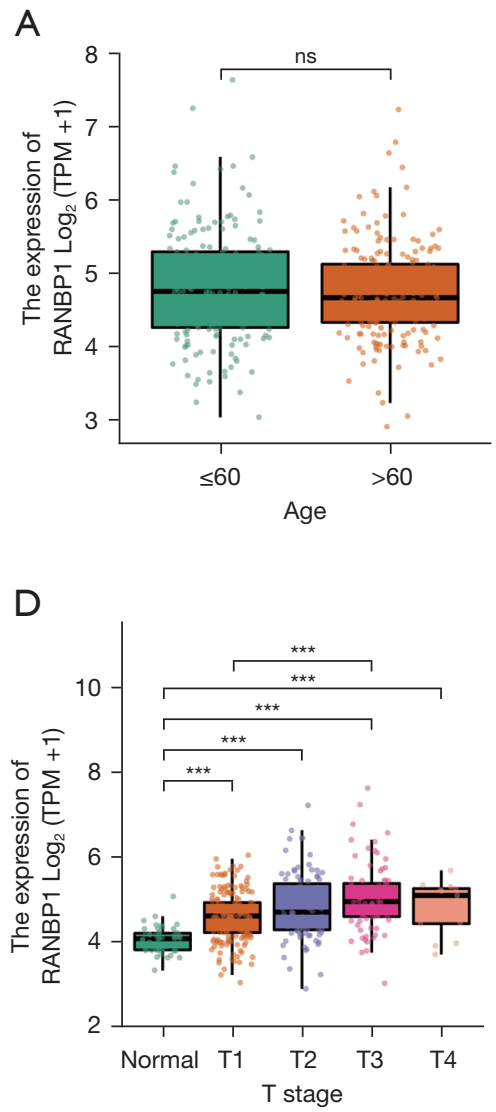

B

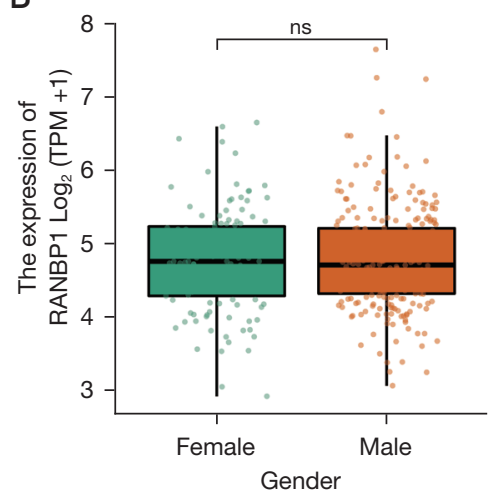

E

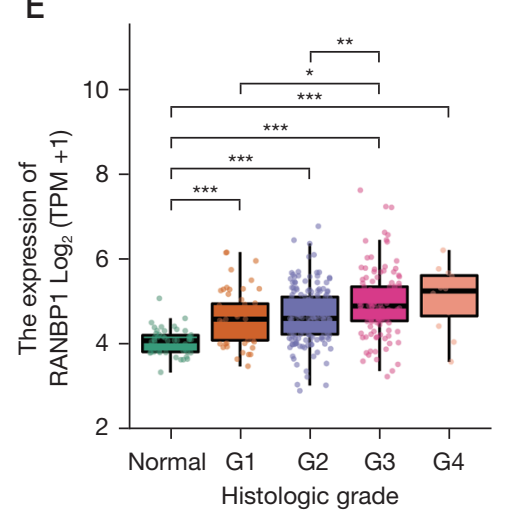

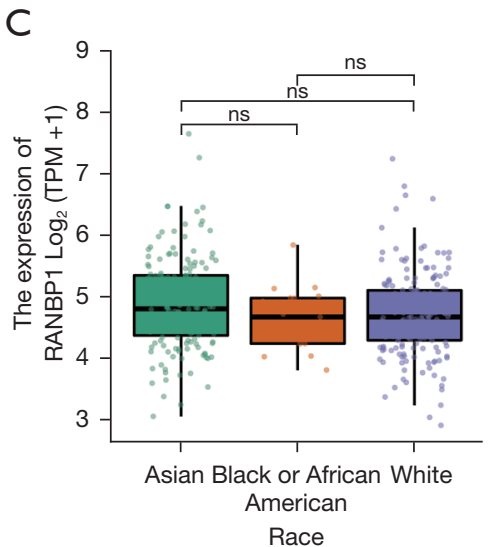

F

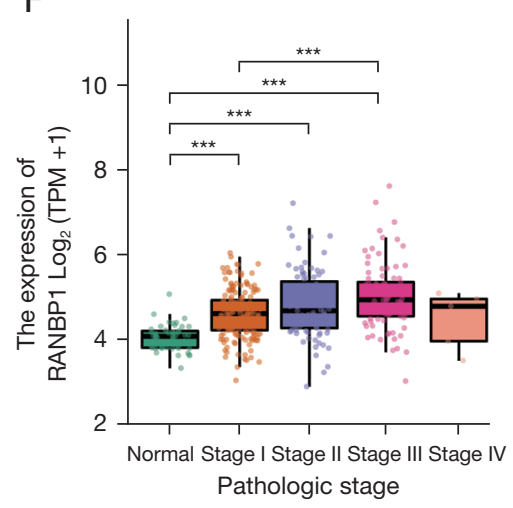

Figure 2 The association with the expression of $R A N B P 1$ and clinical characteristics on TCGA database. (A) RANBP1 expression in HCC tumor tissues among different age groups. (B) RANBP1 expression in HCC tumor tissues for males and females. (C) RANBP1 expression in HCC in different races. Kruskal-Wallis rank sum test was applied in this study. (D) RANBP1 expression associations between normal tissues and different clinicopathologic $\mathrm{T}$ stages. (E) RANBP1 expression associations between normal tissues and different histologic grades. (F) RANBP1 expression associations between normal tissues and different pathologic stages. *, $\mathrm{P}<0.05$; **, $\mathrm{P}<0.01$; ***, $\mathrm{P}<0.001$; ns, not significant. RANBP1, Ran-specific binding protein 1; TCGA, The Cancer Genome Atlas; HCC, hepatocellular carcinoma.

expression group had a shorter OS (HR: 1.68 ; $95 \%$ CI: 1.06-2.64; $\mathrm{P}=0.026)$ and PFI (HR: 1.81; 95\% CI: 1.24-2.65; $\mathrm{P}=0.002$ ) regarding histologic grade (Figure $3 A-3 C$ ), indicating the prognostic value of $R A N B P 1$ in patients with early-stage HCC.

These results proved that elevated $R A N B P 1$ expression was linked to unfavorable outcomes in individuals with HCC.

To further confirm this result, we downloaded survival data from TCGA databases and performed survival analysis to investigate the clinical relevance of RANBP1 expression in patient survival. As shown in Table 2, univariate analysis demonstrated that high $R A N B P 1$ expression was significantly linked to very poor OS (HR: 1.946; 95\% CI: 1.364-2.775; $\mathrm{P}<0.001)$; other clinicopathologic variables associated with poor survival included advanced $\mathrm{T}$ stage (HR: $2.109 ; 95 \%$ CI: $1.47-3.03 ; \mathrm{P}<0.001)$. As shown in Figure $3 D-3 F$, a multivariate Cox regression analysis indicated that $R A N B P 1$ remained independently associated with OS (HR: 1.78; 95\% CI: 1.11-2.89; P=0.018). Similar results were also obtained for PFI (Table S1) and DSS (Table S2) for patients with HCC.

\section{Visualization of the DNA methylation levels of RANBP1 and their prognostic values in $\mathrm{HCC}$}

We employed the MethSurv tool to visualize the DNA CpG methylation levels of RANBP1 (Figure $4 A$ ). Three CpGs loci were selected in the RANBP1 gene, including cg17561788, cg07620854, and cg11684740 (Figure 4B-4D). 
A
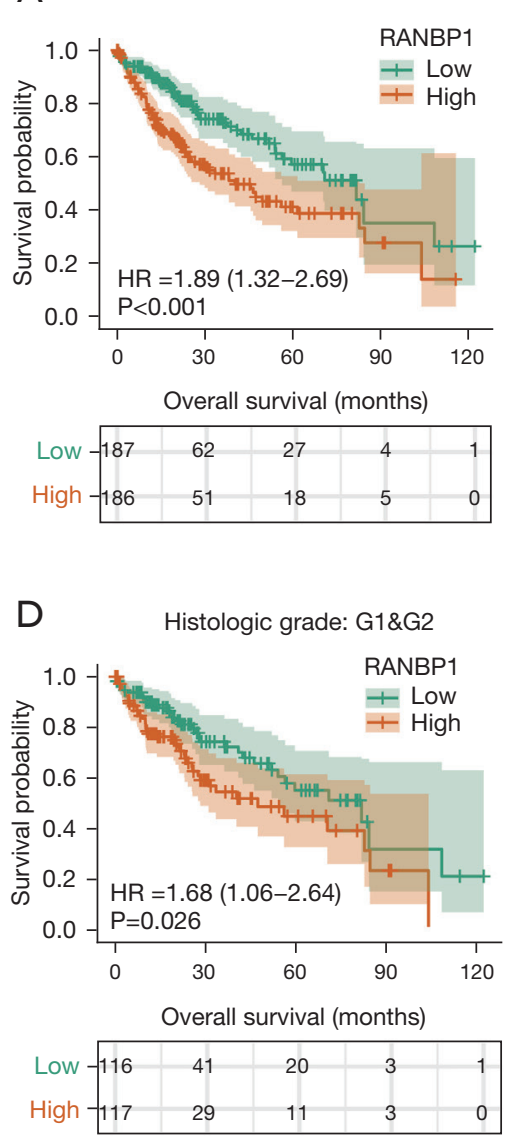

B

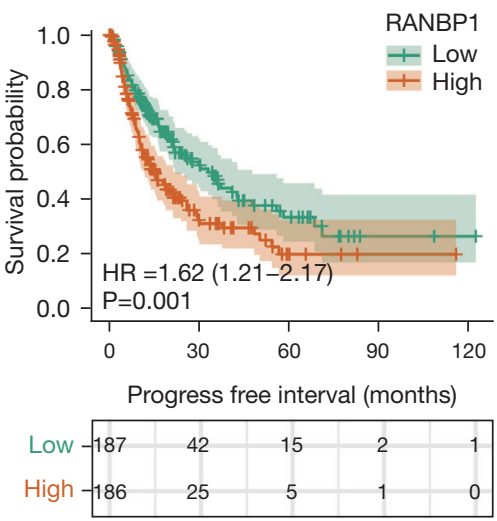

E Histologic grade: G1\&G2

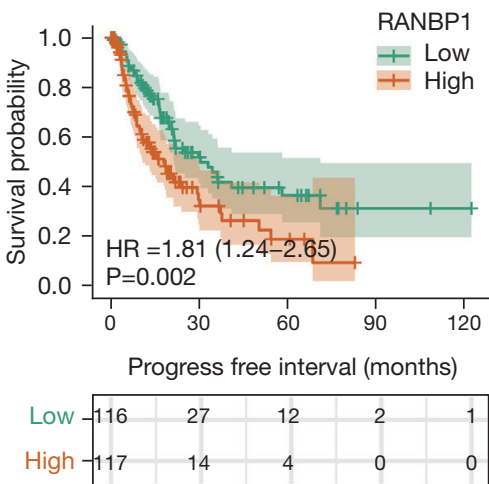

C

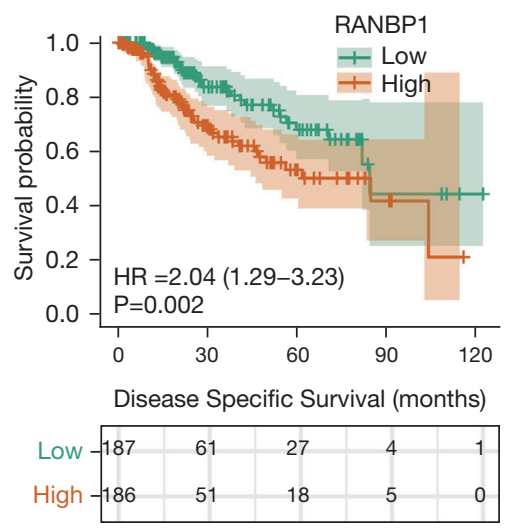

$\mathrm{F}$

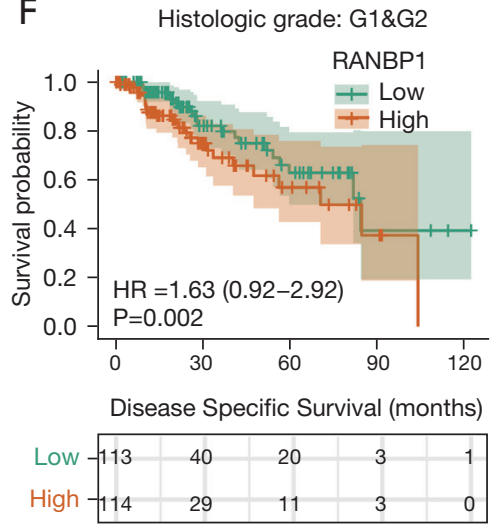

Figure 3 Kaplan-Meier survival analysis of HCC patients with respect to RANBP1 expression, including impact of RANBP1 expression on OS (A), PFI (B), and DSS (C) in HCC patients in TCGA cohort. Kaplan-Meier survival analysis of OS (D), PFI (E), and DSS (F) in HCC patients with low histologic grade subgroup (G1 \& G2). HCC, hepatocellular carcinoma; RANBP1, Ran-specific binding protein 1; OS, overall survival; PFI, progression-free interval; DSS, disease-specific survival; TCGA, The Cancer Genome Atlas.

Furthermore, the prognostic value of the three CpGs was explored. As displayed in Figure 4, all of the three CpGs were associated with survival outcomes. High DNA methylation levels of cg17561788 ( $\mathrm{P}=0.019)$, cg07620854 $(\mathrm{P}<0.001)$, and cg11684740 $(\mathrm{P}<0.001)$ were significantly correlated with very poor OS in HCC patients (Figure $4 B-4 D$ ).

\section{Co-expression network of RANBP1 in HCC and selection of bub genes}

To gain a better understanding of the biological function of RANBP1 in HCC, the function module in linkedOmics was used to analyze the co-expression pattern of $R A N B P 1$ in HCC patients in TCGA database. As illustrated in Figure $5 A$, the volcano plot showed $R A N B P 1$-related different expression genes (DEGs) $(\mathrm{FDR}<0.01)$ with
4580 positively correlated genes (dark red dots) and 4255 negatively correlated genes (dark green dots). Figure 5B,5C shows the heatmaps of the top 50 genes positively and negatively correlated with $R A N B P 1$, respectively. This result implies that $R A N B P 1$ and the positively correlated co-expression genes form a complex regulatory network, which co-regulates the progression of HCC.

Gene Ontology (GO) term annotation illustrated that genes differentially expressed in association with $R A N B P 1$ were located mainly in the endoplasmic reticulum, translational initiation, ribosomal ribonucleic acid (rRNA) metabolic process, ribonucleoprotein complex biogenesis, non-coding ribonucleic acid (ncRNA) processing, and RNA catabolic process, etc. (Figure 5D). Kyoto Encyclopedia of Genes and Genomes (KEGG) pathway analysis illustrated enrichment in ribosome, spliceosome, DNA replication, 
Table 2 Univariate and multivariate Cox proportional hazards analysis of RANBP1 expression and OS for patients with HCC in TCGA cohort

\begin{tabular}{|c|c|c|c|c|}
\hline Characteristics & \multicolumn{2}{|c|}{ Univariate analysis } & \multicolumn{2}{|c|}{ Multivariate analysis } \\
\hline$T$ stage (T2 \& $T 3$ \& $T 4$ vs. $T 1)$ & $2.109(1.469-3.028)$ & $<0.001$ & $0.872(0.118-6.455)$ & 0.893 \\
\hline N stage (N1 vs. N0) & $2.004(0.491-8.181)$ & 0.333 & & \\
\hline M stage (M1 vs. M0) & $4.032(1.267-12.831)$ & 0.018 & $1.726(0.412-7.234)$ & 0.456 \\
\hline Histologic grade (G3 \& G4 vs. G1 \& G2) & $1.120(0.781-1.606)$ & 0.539 & & \\
\hline Residual tumor (R1 \& R2 vs. R0) & $1.571(0.795-3.104)$ & 0.194 & & \\
\hline Age (>60 vs. $\leq 60)$ & $1.248(0.880-1.768)$ & 0.214 & & \\
\hline Gender (male vs. female) & $0.816(0.573-1.163)$ & 0.260 & & \\
\hline Fibrosis Ishak score (1/2 \& $3 / 4 \& 5 / 6$ vs. 0$)$ & $0.779(0.470-1.293)$ & 0.334 & & \\
\hline Adjacent hepatic tissue inflammation (mild \& severe vs. none) & $1.228(0.755-1.997)$ & 0.409 & & \\
\hline Vascular invasion (yes vs. no) & $1.348(0.890-2.042)$ & 0.159 & & \\
\hline RANBP1 (high vs. low) & $1.946(1.364-2.775)$ & $<0.001$ & $1.788(1.107-2.888)$ & 0.018 \\
\hline
\end{tabular}

$\mathrm{Cl}$, confidence interval; HR, hazard ratio; RANBP1, Ran-specific binding protein 1.

RNA transport, ribosome biogenesis in eukaryotes, pyrimidine metabolism, RNA polymerase, cell cycle, etc. (Figure 5E).

To further validate the co-regulated relationship of the top 100 positively related genes of RANBP1 in HCC, a PPI network was established using the STRING website (Figure 5F). Then, 19 key genes were identified and screened using the MCODE plugin of Cytoscape software, in which the small nuclear ribonucleoprotein D1 polypeptide (SNRPD1) gene (yellow color) was most strongly associated with the RANBP1 expression level (Figure $5 G$ ).

\section{RANBP1-related signaling pathways based on GSEA}

To explore the potential signaling pathways activated in HCC, we conducted GSEA between the low and the high RANBP1 expression data set. As shown in Table 3, significant differences (FDR $<0.05$, normalized $\mathrm{P}<0.05$ ) in the enrichment of the Molecular Signatures Database (MSigDB) Collection (c5. all. v7.0. symbols and h. all. v7.0. symbols) of the diverse cascades were observed. We chose the most significant signaling pathways on the basis of NES (Figure 6 and Table 3). Figure 6 shows that GO cell signaling by Wingless and Int-1 (Wnt), GO chromatin assembly or disassembly, GO DNA binding transcription activator activity, GO immune response regulating cell surface receptor signaling pathway, GO immunoglobulin production, GO microtubule cytoskeleton organization, GO organelle fission, GO DNA repair, and G2M checkpoint are differentially enriched in the RANBP1 high expression phenotype.

\section{The correlation between $R A N B P 1$ expression and immune infiltration}

We then investigated whether RANBP1 expression was related to immune invasion levels (generated by ssGSEA) in HCC using Spearman correlation. As shown in Figure 7, the infiltrating levels of T helper cell type-1 (Th1), T follicular helper cells (Tfh), and NK CD56bright cells were significantly and positively correlated with $R A N B P 1$ expression. On the contrary, T helper cell type-17 (Th17), dendritic cells (DCs), and neutrophil infiltration levels were significantly and negatively correlated with $R A N B P 1$ expression. Meanwhile, the infiltrating levels of Th2 cells, Tfh, and NK CD56bright cells were significantly higher in 

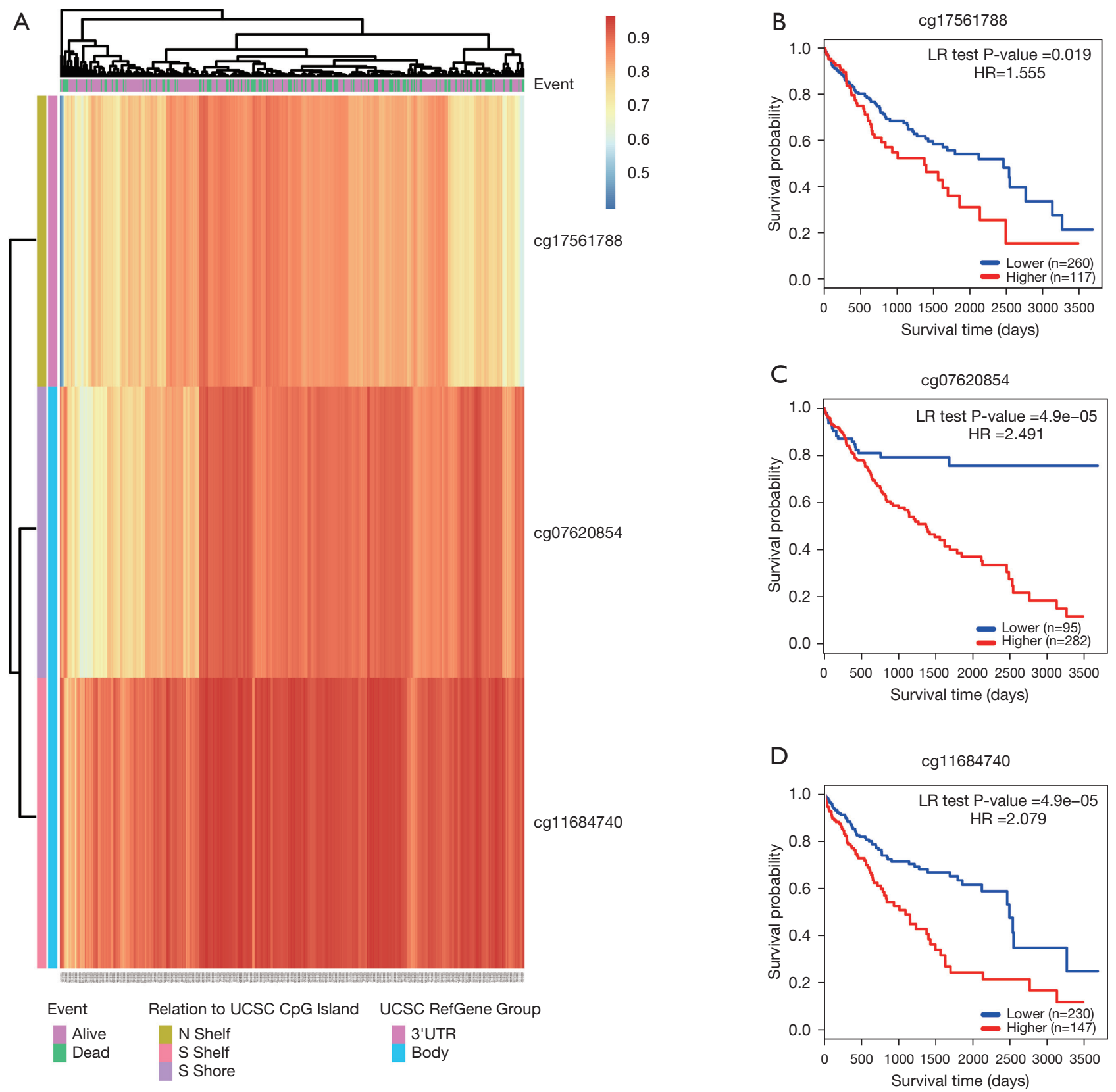

Figure 4 DNA methylation sites of RANBP1 and their prognostic values in HCC. (A) The DNA methylation clustered expression of RANBP1. OS curve of cg17561788 (B), cg07620854 (C), and cg11684740 (D) in HCC patients in MethSurv (https://biit.cs.ut.ee/ methsurv/). DNA, desoxyribonucleic acid; RANBP1, Ran-specific binding protein 1; HCC, hepatocellular carcinoma; OS, overall survival.

the high-RANBP1 expression samples compared to the low expression ones, and the infiltrating levels of Th17 cells, neutrophils, and DCs were significantly lower in highRANBP1 expression samples compared with low expression ones using the Wilcoxon rank sum test.

\section{Discussion}

The RANBP1 was a partner of Ras-related nuclear protein (Ran), with the function that regulates diverse processes as a versatile GTPase $(28,29)$. Noteworthily, a growing 
A

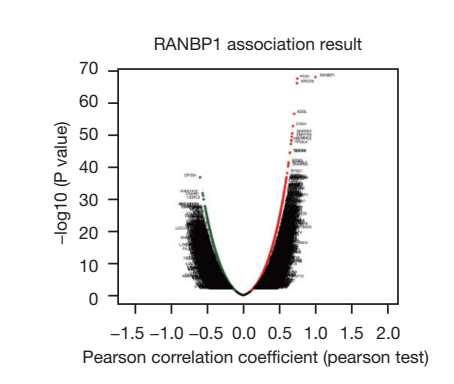

D

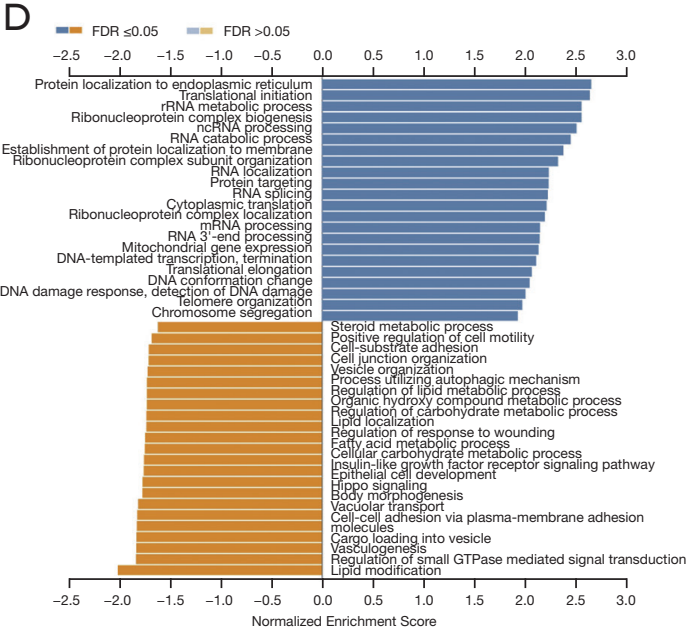

$\mathrm{F}$

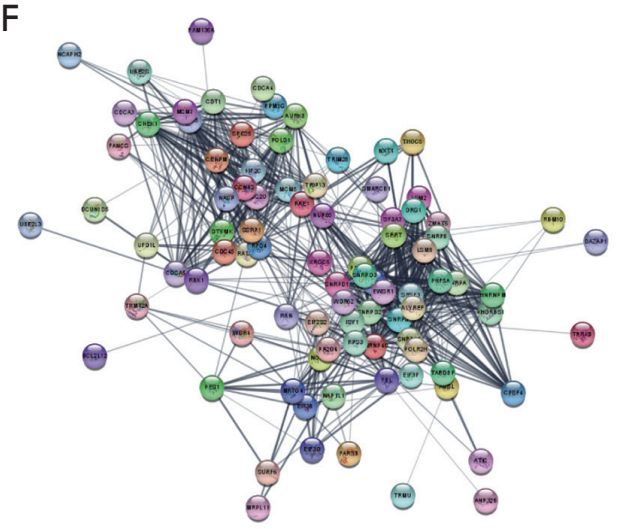

B

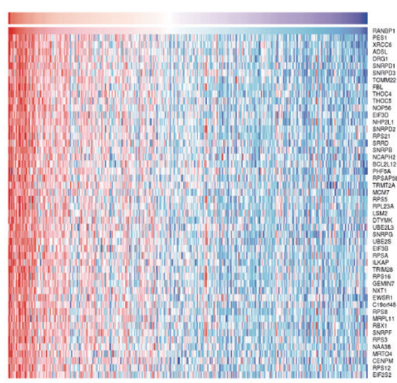

$\mathrm{E}$

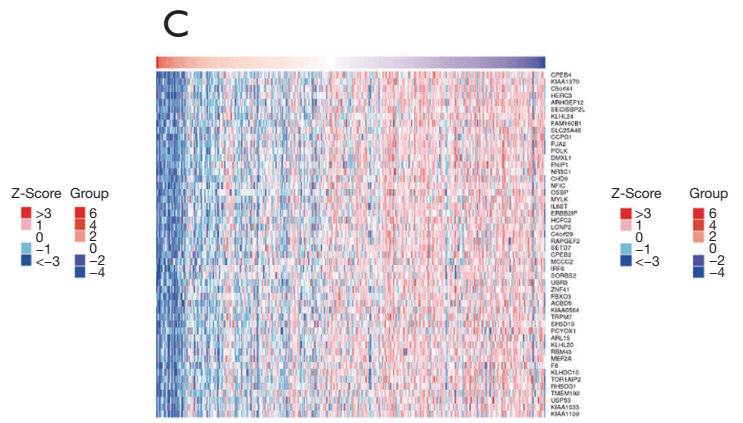

$=\mathrm{FDR} \leq 0.05 \quad=\mathrm{FDR}>0.05$

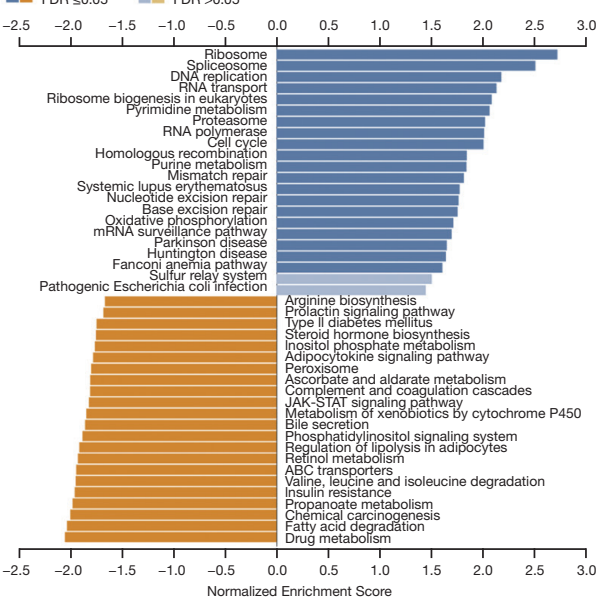

G

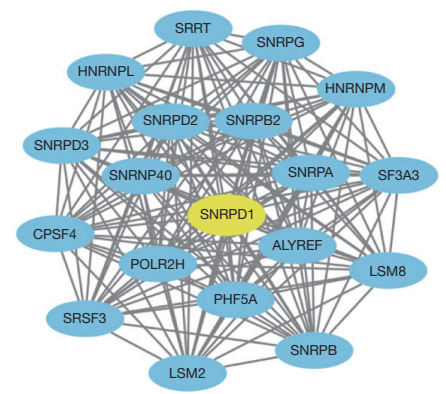

Figure 5 Co-expression genes and protein-protein interaction network associated with RANBP1 expression in HCC. (A) Volcano plot showing the positively and negatively correlated genes in LIHC). (B,C) The top 50 positively and negatively correlated genes with RANBP1 in heat maps. (D) Significant enriched GO annotations of RANBP1 in LIHC. (E) Significant enriched KEGG pathways of RANBP1 in LIHC. (F) The PPI network of RANBP1 expression. (G) The hub genes of RANBP1 co-expression network. RANBP1, Ran-specific binding protein 1; HCC, hepatocellular carcinoma; LIHC, Liver Hepatocellular Carcinoma; PPI, protein-protein interaction; SNRPD1, small nuclear ribonucleoprotein D1 polypeptide; SNRPD2, small nuclear ribonucleoprotein D2 polypeptide; SNRPD3, small nuclear ribonucleoprotein D3 polypeptide; SNRPG, small nuclear ribonucleoprotein polypeptide G; SNRPB, small nuclear ribonucleoprotein polypeptides B And B1; SNRPB2, small nuclear ribonucleoprotein polypeptides B2; SNRPA, small nuclear ribonucleoprotein polypeptide A; SRRT, serrate RNA effector molecule; SRSF3, serine and arginine rich splicing factor 3; SNRNP40, small nuclear ribonucleoprotein U5 subunit 40; POLR2H, RNA polymerase II, I And III subunit H; LSM2, LSM2 homolog, U6 small nuclear RNA and MRNA degradation associated; LSM8, LSM8 homolog, U6 small nuclear RNA associated; HNRNPL, heterogeneous nuclear ribonucleoprotein L; CPSF4, cleavage and polyadenylation specific factor 4; HNRNPM, heterogeneous nuclear ribonucleoprotein M; SF3A3, splicing factor 3a subunit 3; ALYREF, Aly/REF export factor; PHF5A, PHD finger protein 5A. 
Table 3 Gene sets enriched in high phenotype

\begin{tabular}{|c|c|c|c|c|}
\hline MSigDB collection & Gene set name & NES & p.adjust & FDR \\
\hline \multirow{6}{*}{ c5.all.v7.0.symbols.gmt } & GO_CHROMATIN_ASSEMBLY_OR_DISASSEMBLY & 2.33 & 0.018 & 0.012 \\
\hline & GO_DNA_BINDING_TRANSCRIPTION_ACTIVATOR_ACTIVITY & 2.061 & 0.018 & 0.012 \\
\hline & $\begin{array}{l}\text { GO_IMMUNE_RESPONSE_REGULATING_CELL_SURFACE_ } \\
\text { RECEPTOR_SIGNALING_PATHWAY }\end{array}$ & 2.288 & 0.018 & 0.012 \\
\hline & GO_MICROTUBULE_CYTOSKELETON_ORGANIZATION & 2.067 & 0.018 & 0.012 \\
\hline & GO_ORGANELLE_FISSION & 2.402 & 0.018 & 0.012 \\
\hline & GO_DNA_REPAIR & 2.271 & 0.018 & 0.012 \\
\hline
\end{tabular}

Gene sets with p.adjust $<0.05$ and FDR $<0.25$ are considered as significant. NES, normalized enrichment score; FDR, false discovery rate; MSigDB, Molecular Signatures Database.

number of studies found that the genes encoding Ran and its partners are abnormally expressed in cancer cells (30-33). Without exception, RANBP1 was also found to be abnormally expressed in many cancers (34). Therefore, we paid attention to the quantity of RANBP1 and its potential for prognosis in HCC.

In the present study, we found that $R A N B P 1$ expression was elevated in pan-cancer and especially in HCC. Our study has provided a deeper understanding of the relationship between $R A N B P 1$ expression and clinical features, prognosis, immune cell infiltration, and key genes in HCC. Our results demonstrate that RANBP1 expression was linked to the patient's T stage, pathologic stage, histologic grade, and survival analysis indicated that $R A N B P 1$ might serve as a potential prognostic and prognostic marker in early-stage HCC.

As we know, the expression and function of genes could be affected by DNA methylation. Some studies demonstrate that the methylation status of associated genes is associated with cell proliferation and migration (35), and therefore we also analyzed the methylation status of $R A N B P 1$ promotors in HCC. At present, we showed that RANBP1 was up-regulated in HCC and the high methylation level of the three CpG sites of RANBP1 was associated with poor outcomes. However, this is different from the wellknown theory that DNA methylation in the promoter region often leads to gene silencing (36). One possible conjecture is that $R A N B P 1$ methylation status might be regulated by a complex network in the tumorigenesis of HCC. Further study is needed to elucidate the molecular mechanisms. To assess the signaling network in controlling abnormal expression of $R A N B P 1$, we further investigated the co-expression network. SNRPD1 was found to be most strongly associated with $R A N B P 1$ expression, and several studies reported that the overexpressed SNRPD1 was significantly associated with poor outcomes in LUAD (37). Moreover, these co-expression genes are involved in DNA replication and the RNA transport process, which is consistent with the function of $R A N B P 1$. All findings indicate that $R A N B P 1$ is regulated by a complex network and associated with a poor prognosis of HCC.

The GO and KEGG enrichment analysis, such as translational initiation, ribosome, spliceosome, DAN replication, RNA transport, and cell cycle indicated that $R A N B P 1$ was relevant to cell growth and proliferation. A GSEA analysis showed roles in G2M-checkpoint, chromatin assembly, DNA binding transcription, DNA repair, and Wnt cell signaling. This is consistent with GO and KEGG enrichment founding. The alteration of the cell cycle has been acknowledged as one of the hallmarks of cancer. The G2M-checkpoint mediates G2-M cell cycle transition (38). Wnt cell signaling is pivotal for proliferation, as well as the maintenance of cancer stem cell-like traits of diverse cancer cells. Also, several studies found that G2M-checkpoint (39), Wnt cell signaling (40), and DNA repair (41) were closely correlated with the initiation and development of tumor cells. It indicated that the high expression of RANBP1 may affect cell growth and proliferation through G2M checkpoints and Wnt cell signaling. These findings further support the relationship between RANBP1 and 


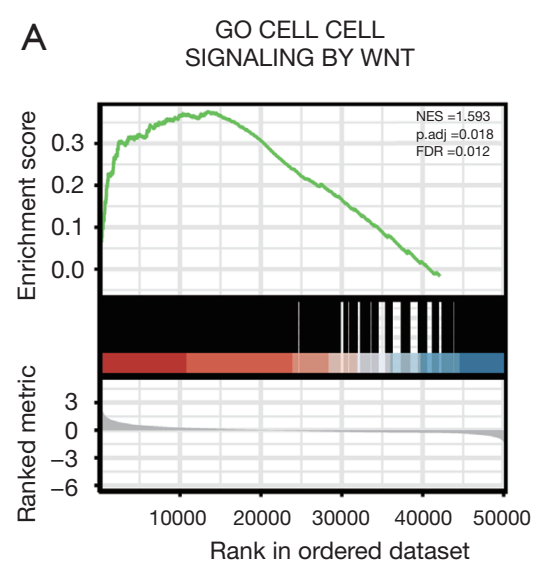

GO IMMUNE RESPONSE

D REGULATING CELL SURFACE RECEPTOR SIGNALING PATHWAY

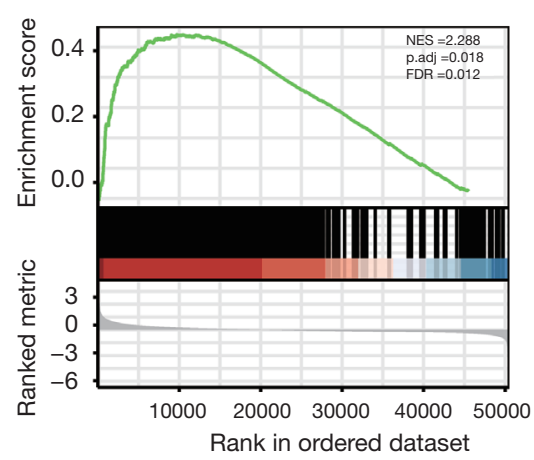

G GO ORGANELLE FISSION

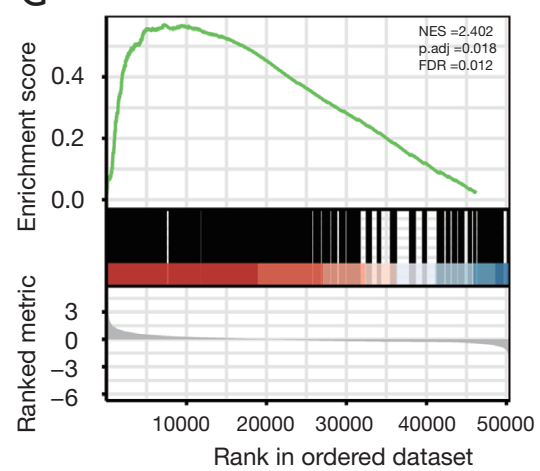

B GO CHROMATIN ASSEMBLY OR DISASSEMBLY
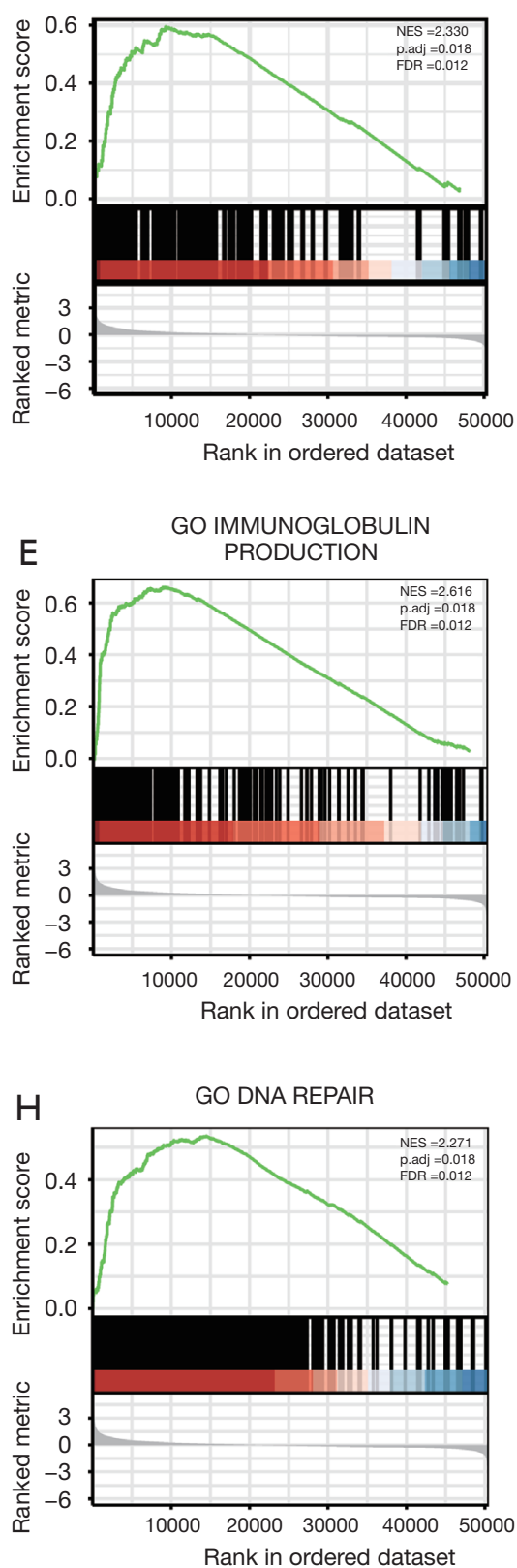

\section{GO DNA BINDING TRANSCRIPTION ACTIVATOR ACTIVITY}

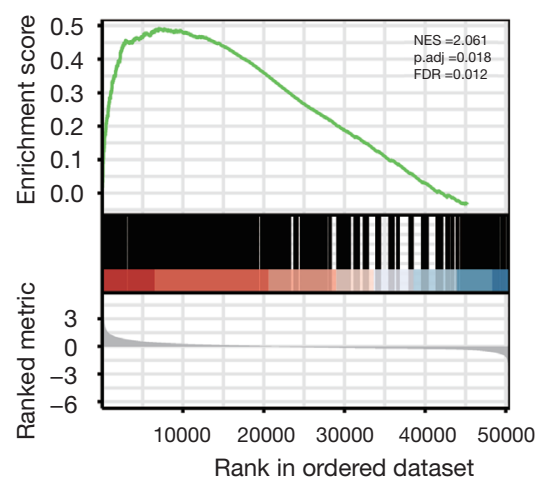

GO MICROTUBULE
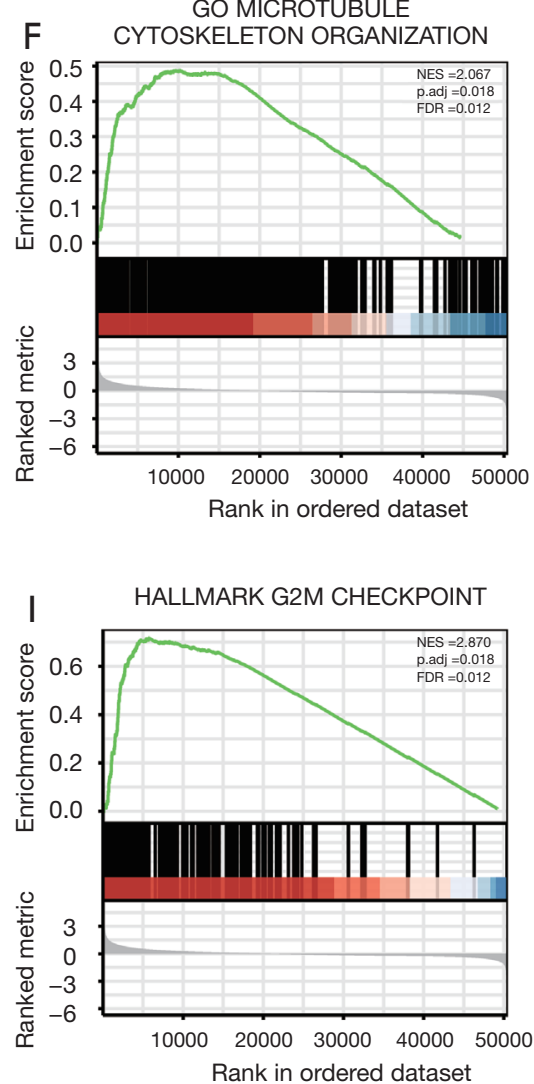

Figure 6 Enrichment plots from GSEA. GSEA results showing signaling by Wnt (A), chromatin assembly or disassembly (B), DNA binding transcription activator activity (C), cell surface receptor signaling pathway (D), immunoglobulin production (E), microtubule cytoskeleton organization (F), organelle fission (G), DNA repair (H), G2M-checkpoint (I). The GSEA software was used to calculate enrichment levels. GSEA, gene set enrichment analysis; DNA, desoxyribonucleic acid; ES, enrichment score; NES, normalized ES; p. adj, adjust P value; FDR, false discovery rate. 
A

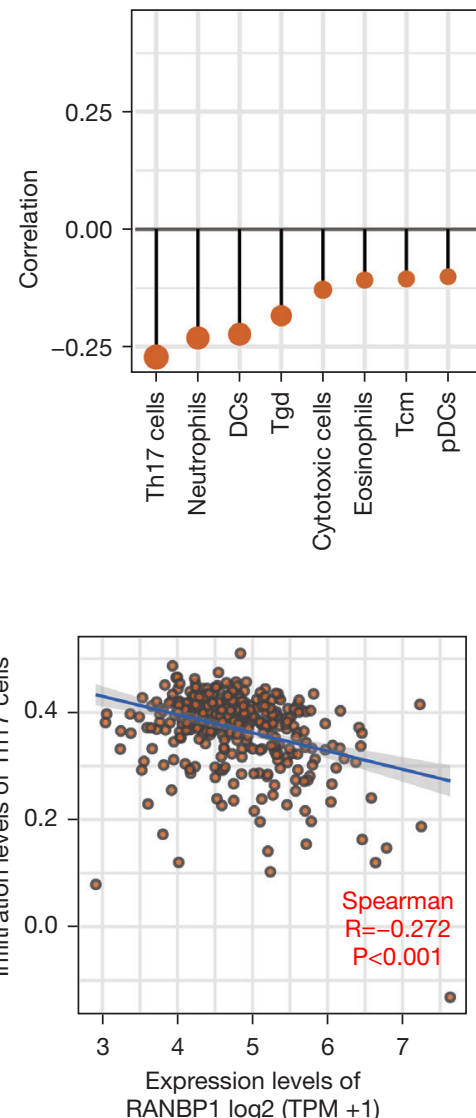

E

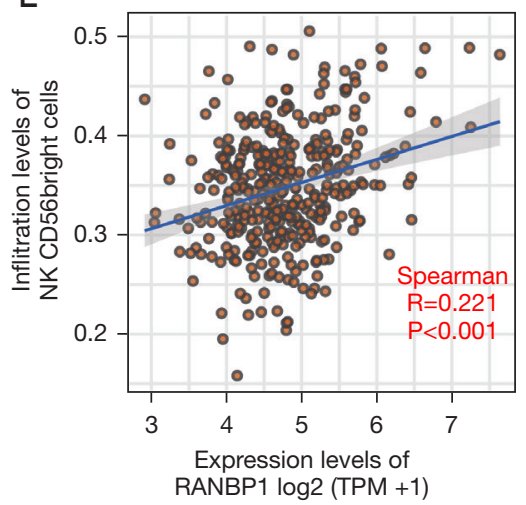

$\mathrm{H}$

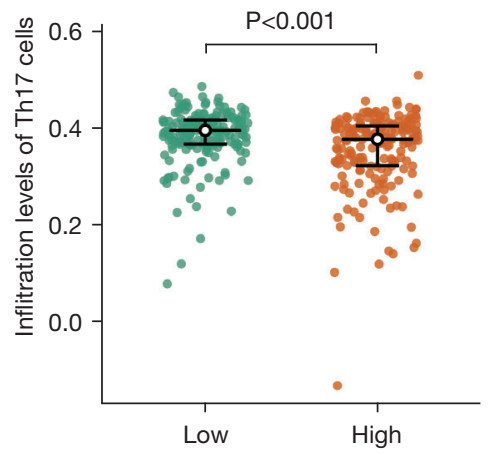

C

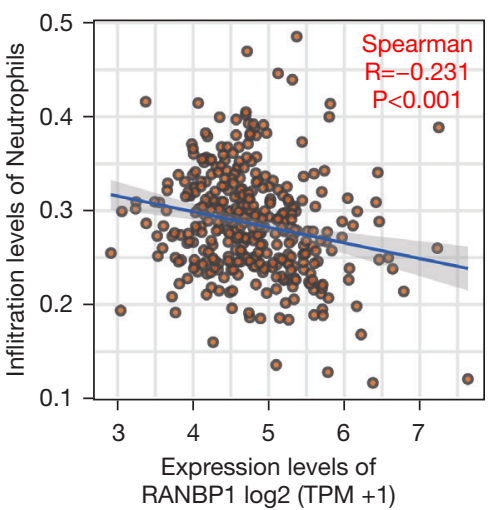

\section{$\mathrm{F}$}

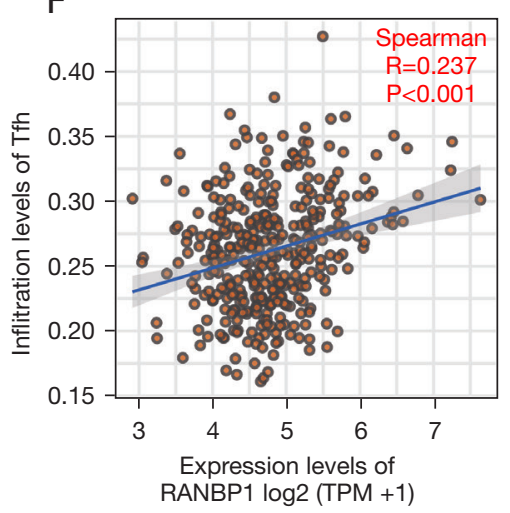

I

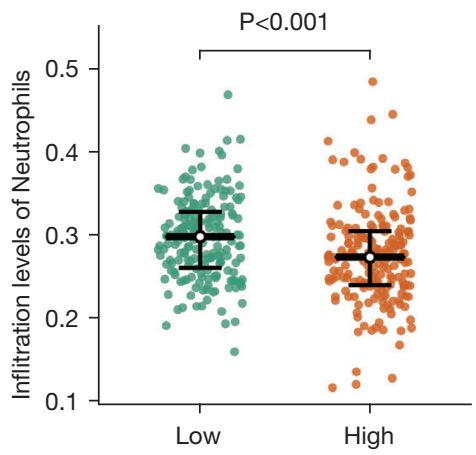

$P$ value

0.75

0.50

0.25

0.00

Correlation

o 0.1

O 0.2

○े 0.3
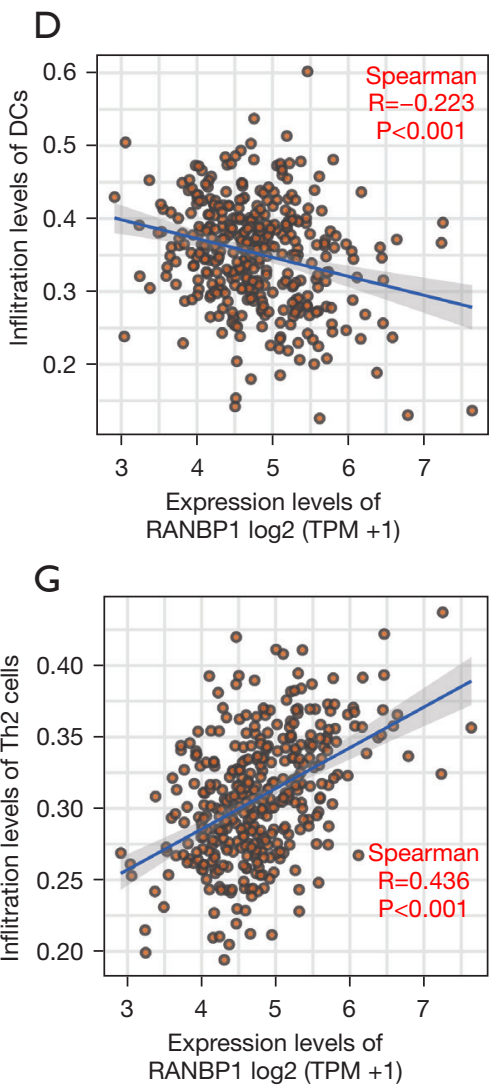

J

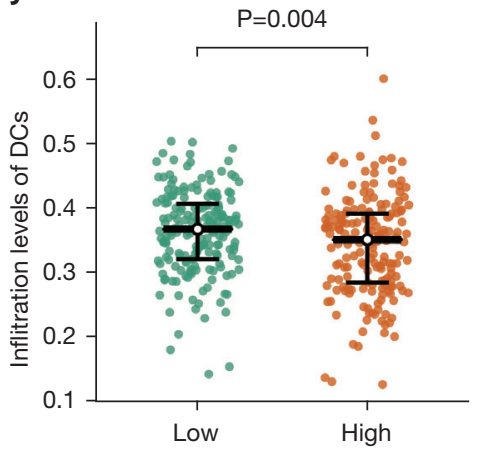



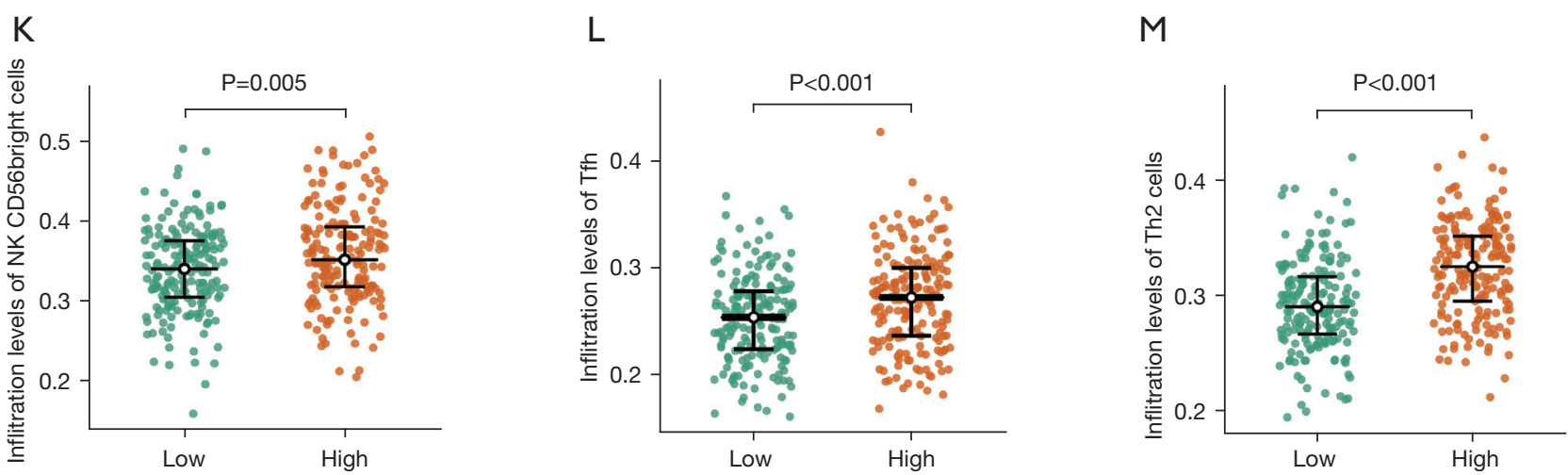

Figure 7 Expression level of RANBP1 was associated with immune infiltration in tumor microenvironment. (A) The lollipop chart shows the correlation between the RANBP1 expression level and relative quantities of 24 types of immune cells in HCC patients. The expression level (TPM) of RANBP1 is significantly and positively correlated with infiltrating levels of NK CD56bright cells (E,K), Tfh cells (F,L), Th2 cells $(\mathrm{G}, \mathrm{M})$, and significantly and negatively correlated with infiltrating levels of Th17 cells (B,H), neutrophils (C,I), and DCs (D,J) in HCC. RANBP1, Ran-specific binding protein 1; Th17, type-17 T helper cells; Th1, type-1 T helper cells; Th2, type-2 T helper cells; DCs, dendritic cells; aDCs, activated dendritic cells; iDCs, immature dendritic cells; Th, T helper cells; Treg, regulatory T cells; Tgd, gamma delta T cells; Tcm, central memory T cells; Tem, effector memory T cells; Tfh, follicular helper T cells.

tumorigenesis.

In fact, RANBP1 is a component of the cell cycleregulated Ran network in mammalian cells, where the quantities are highest during the G2 and $M$ phases (42). Several observations indicate that RanBP1 contributes to regulate the function of the mitotic apparatus: RanBP1 inactivation yields hyperstable MTs and induces apoptosis during mitosis (43). It has previously been found that RanBP1 down-regulation yields hyperstable mitotic microtubules (MTs) and induction of apoptosis by changing the distribution of hepatoma up-regulated protein (HURP), which is viewed as a potential oncogene (15). It is possible that up-regulated RANBP1 results in inadequate apoptosis and promotes tumorigenesis and contributes to a poor outcome.

Exosomes are now considered as important mediators of host anti-tumor immune response as well as tumor cell immune escape. HCC-derived exosomes have been shown to attenuate the cytotoxicity of T-cells and NK cells, and promote the immuno-suppressive M2 macrophages, N2 neutrophils, and Bregs (44). Meanwhile, immune cells are also known as key players in the HCC microenvironment and show complicated crosstalk with cancer cells (45). A noteworthy conclusion of this work involves the relationship between the expression of RANBP1 and the level of immune invasion in HCC. The ssGSEA analysis revealed a significant relationship between $R A N B P 1$ expression and the infiltration levels of Th2 cells, Tfh cells, CD56 bright cells, Th17 cells, DCs, and neutrophils in HCC, indicating an important effect of $R A N B P 1$ in regulating the tumor immune microenvironment of HCC.

We found that the proportions of Th2 cells had significant positive correlations with RANBP1, whereas Th17 cells had significant negative correlations. A large number of studies found that strong Th2 infiltration was linked to poor outcomes in ovarian cancer (46), pancreatic cancer (47), and gastric cancer (48). It has also been reported (49) that Th2 cells may be involved in aggressive tumors through activation of $\mathrm{B}$ cells or production of immunosuppressive cytokine interleukin 10 (IL-10) (49). In our study, we found that the high expression of RANBP1 showed a positive correlation with infiltration levels of Th2 cells. The correlation may indicate the potential regulating role of RANBP1 in tumor-associated B cells and cytokine production. Moreover, previous studies have shown that the infiltration levels of Th17 cells were related to better survival in ovarian cancer (50), oesophageal cancer (51), and gastric cancer (52). However, the higher expression of RANBP1 and the lower infiltration of Th17 cells also indicated that high $R A N B P 1$ expression might be a poor prognostic factor. Collectively, these findings show that $R A N B P 1$ plays a crucial role in the regulation and recruitment of immune infiltrating cells in HCC. However, the underlying mechanism of RANBP1 and immune cells 
needs to be further elucidated.

In conclusion, we demonstrated that $R A N B P 1$ plays a pivotal role in the molecular mechanism of HCC tumorigenesis, that high expression of $R A N B P 1$ is a prospective prognostic molecular marker for very poor survival in HCC, and that G2M-checkpoint, Wnt cell signaling, and DNA repair may be the key pathways for RANBP1 regulation in HCC. Also, RANBP1 may have an essential impact on immune infiltration and has the potential to serve as a prospective candidate diagnostic biomarker, prognostic factor, and therapeutic target of HCC. Nevertheless, we are aware of some limitations of our work, including that more clinical factors need to be considered to fully clarify the exact role of RANBP1 in the development of HCC, and that there is no experimental proof of the mechanism between $R A N B P 1$ and immune cells in HCC. Future work needs to focus on the precise mechanism of the association between high expression $R A N B P 1$ with poor outcome and the regulatory mechanism between $R A N B P 1$ and immune cells.

\section{Acknowledgments}

We thank all the investigators who shared their highthroughput expression data.

Funding: The project was supported by National Natural Science Foundation of China (Grant No. 61901072), Natural Science Foundation of Chongqing (Grant No. cstc2019jcyj-msxmX0250) and Chongqing Education Commission Foundation (Grant No. KJQN201800620).

\section{Footnote}

Reporting Checklist: The authors have completed the REMARK reporting checklist. Available at https://dx.doi. org/10.21037/jgo-21-541

Conflicts of Interest: All authors have completed the ICMJE uniform disclosure form (available at https://dx.doi. org/10.21037/jgo-21-541). The authors have no conflicts of interest to declare.

Ethical Statement: The authors are accountable for all aspects of the work in ensuring that questions related to the accuracy or integrity of any part of the work are appropriately investigated and resolved. This article does not contain any studies involving human participants or animals. Since all datasets included in the present study were downloaded from TCGA and GTEx, additional approval by an ethics committee was not needed. The study was conducted in accordance with Declaration of Helsinki (as revised in 2013).

Open Access Statement: This is an Open Access article distributed in accordance with the Creative Commons Attribution-NonCommercial-NoDerivs 4.0 International License (CC BY-NC-ND 4.0), which permits the noncommercial replication and distribution of the article with the strict proviso that no changes or edits are made and the original work is properly cited (including links to both the formal publication through the relevant DOI and the license). See: https://creativecommons.org/licenses/by-nc-nd/4.0/.

\section{References}

1. Henley SJ, Ward EM, Scott S, et al. Annual report to the nation on the status of cancer, part I: National cancer statistics. Cancer 2020;126:2225-49.

2. Siegel RL, Miller KD, Jemal A. Cancer statistics, 2019. CA Cancer J Clin 2019;69:7-34.

3. Lee J, Cho Y, Park S, et al. Skeletal Muscle Depletion Predicts the Prognosis of Patients With Hepatocellular Carcinoma Treated With Radiotherapy. Front Oncol 2019;9:1075.

4. Lu SD, Li L, Liang XM, et al. Updates and advancements in the management of hepatocellular carcinoma patients after hepatectomy. Expert Rev Gastroenterol Hepatol 2019;13:1077-88.

5. Njei B, Rotman Y, Ditah I, et al. Emerging trends in hepatocellular carcinoma incidence and mortality. Hepatology 2015;61:191-9.

6. Zhao J, Hou Y, Yin C, et al. Upregulation of histamine receptor $\mathrm{H} 1$ promotes tumor progression and contributes to poor prognosis in hepatocellular carcinoma. Oncogene 2020;39:1724-38.

7. Xie X, Yu Y. Prognosis value of liver stiffness measurements by 2D-SWE in primary HBV-positive hepatocellular carcinoma following radiofrequency ablation. Transl Cancer Res 2020;9:2518-26.

8. Wang Y, Wang G, Tan X, et al. MT1G serves as a tumor suppressor in hepatocellular carcinoma by interacting with p53. Oncogenesis 2019;8:67.

9. Ge PL, Li SF, Wang WW, et al. Prognostic values of immune scores and immune microenvironment-related 
genes for hepatocellular carcinoma. Aging (Albany NY) 2020;12:5479-99.

10. Long J, Wang A, Bai Y, et al. Development and validation of a TP53-associated immune prognostic model for hepatocellular carcinoma. EBioMedicine 2019;42:363-74.

11. Koyama M, Matsuura Y. An allosteric mechanism to displace nuclear export cargo from CRM1 and RanGTP by RanBP1. EMBO J 2010;29:2002-13.

12. Ly TK, Wang J, Pereira R, et al. Activation of the Ran GTPase is subject to growth factor regulation and can give rise to cellular transformation. J Biol Chem 2010;285:5815-26.

13. Amato R, Scumaci D, D'Antona L, et al. Sgk1 enhances RANBP1 transcript levels and decreases taxol sensitivity in RKO colon carcinoma cells. Oncogene 2013;32:4572-8.

14. Li HY, Ng WP, Wong CH, et al. Coordination of chromosome alignment and mitotic progression by the chromosome-based Ran signal. Cell Cycle 2007;6:1886-95.

15. Tedeschi A, Ciciarello M, Mangiacasale R, et al. RANBP1 localizes a subset of mitotic regulatory factors on spindle microtubules and regulates chromosome segregation in human cells. J Cell Sci 2007;120:3748-61.

16. Zhang Q, Huang S, Luo H, et al. Eight-plex iTRAQ labeling and quantitative proteomic analysis for human bladder cancer. Am J Cancer Res 2017;7:935-45.

17. Vivian J, Rao AA, Nothaft FA, et al. Toil enables reproducible, open source, big biomedical data analyses. Nat Biotechnol 2017;35:314-6.

18. Lian Q, Wang S, Zhang G, et al. HCCDB: A Database of Hepatocellular Carcinoma Expression Atlas. Genomics Proteomics Bioinformatics 2018;16:269-75.

19. Uhlén M, Fagerberg L, Hallström BM, et al. Proteomics. Tissue-based map of the human proteome. Science 2015;347:1260419.

20. Liu J, Lichtenberg T, Hoadley KA, et al. An Integrated TCGA Pan-Cancer Clinical Data Resource to Drive HighQuality Survival Outcome Analytics. Cell 2018;173:400416.e11.

21. Li Y, Jiao Y, Fu Z, et al. High miR-454-3p expression predicts poor prognosis in hepatocellular carcinoma. Cancer Manag Res 2019;11:2795-802.

22. Modhukur V, Iljasenko T, Metsalu T, et al. MethSurv: a web tool to perform multivariable survival analysis using DNA methylation data. Epigenomics 2018;10:277-88.

23. Vasaikar SV, Straub P, Wang J, et al. LinkedOmics: analyzing multi-omics data within and across 32 cancer types. Nucleic Acids Res 2018;46:D956-63.

24. Szklarczyk D, Morris JH, Cook H, et al. The STRING database in 2017: quality-controlled protein-protein association networks, made broadly accessible. Nucleic Acids Res 2017;45:D362-8.

25. Subramanian A, Tamayo P, Mootha VK, et al. Gene set enrichment analysis: a knowledge-based approach for interpreting genome-wide expression profiles. Proc Natl Acad Sci U S A 2005;102:15545-50.

26. Yu G, Wang LG, Han Y, et al. clusterProfiler: an R package for comparing biological themes among gene clusters. OMICS 2012;16:284-7.

27. Hänzelmann S, Castelo R, Guinney J. GSVA: gene set variation analysis for microarray and RNA-seq data. BMC Bioinformatics 2013;14:7.

28. Bindea G, Mlecnik B, Tosolini M, et al. Spatiotemporal dynamics of intratumoral immune cells reveal the immune landscape in human cancer. Immunity 2013;39:782-95.

29. Reich M, Liefeld T, Gould J, et al. GenePattern 2.0. Nat Genet 2006;38:500-1.

30. Plafker K, Macara IG. Facilitated nucleocytoplasmic shuttling of the Ran binding protein RanBP1. Mol Cell Biol 2000;20:3510-21.

31. Görlich D, Seewald MJ, Ribbeck K. Characterization of Ran-driven cargo transport and the RanGTPase system by kinetic measurements and computer simulation. EMBO J 2003;22:1088-100.

32. Sheng C, Qiu J, Wang Y, et al. Knockdown of Ran GTPase expression inhibits the proliferation and migration of breast cancer cells. Mol Med Rep 2018;18:157-68.

33. Ning J, Liu W, Zhang J, et al. Ran GTPase induces EMT and enhances invasion in non-small cell lung cancer cells through activation of PI3K-AKT pathway. Oncol Res 2013;21:67-72.

34. Rensen WM, Mangiacasale R, Ciciarello $M$, et al. The GTPase Ran: regulation of cell life and potential roles in cell transformation. Front Biosci 2008;13:4097-121.

35. Shan J, Dsouza SP, Bakhru S, et al. TNRC9 downregulates BRCA1 expression and promotes breast cancer aggressiveness. Cancer Res 2013;73:2840-9.

36. Yang X, Han H, De Carvalho DD, et al. Gene body methylation can alter gene expression and is a therapeutic target in cancer. Cancer Cell 2014;26:577-90.

37. Yi M, Li T, Qin S, et al. Identifying Tumorigenesis and Prognosis-Related Genes of Lung Adenocarcinoma: Based on Weighted Gene Coexpression Network Analysis. Biomed Res Int 2020;2020:4169691.

38. Lock RB, Ross WE. Inhibition of p34cdc2 kinase activity by etoposide or irradiation as a mechanism of G2 arrest in Chinese hamster ovary cells. Cancer Res 1990;50:3761-6. 
39. Oshi M, Newman S, Tokumaru Y, et al. High G2M Pathway Score Pancreatic Cancer is Associated with Worse Survival, Particularly after Margin-Positive (R1 or R2) Resection. Cancers (Basel) 2020;12:2871.

40. Taciak B, Pruszynska I, Kiraga L, et al. Wnt signaling pathway in development and cancer. J Physiol Pharmacol 2018.

41. Cortez D. Replication-Coupled DNA Repair. Mol Cell 2019;74:866-76.

42. Ciciarello M, Roscioli E, Di Fiore B, et al. Nuclear reformation after mitosis requires downregulation of the Ran GTPase effector RanBP1 in mammalian cells. Chromosoma 2010;119:651-68.

43. Rensen WM, Roscioli E, Tedeschi A, et al. RanBP1 downregulation sensitizes cancer cells to taxol in a caspase3-dependent manner. Oncogene 2009;28:1748-58.

44. Han Q, Zhao H, Jiang Y, et al. HCC-Derived Exosomes: Critical Player and Target for Cancer Immune Escape. Cells 2019;8:558.

45. Sia D, Jiao Y, Martinez-Quetglas I, et al. Identification of an Immune-specific Class of Hepatocellular Carcinoma, Based on Molecular Features. Gastroenterology 2017;153:812-26.

46. Kusuda T, Shigemasa K, Arihiro K, et al. Relative expression levels of Th1 and Th2 cytokine mRNA are independent prognostic factors in patients with ovarian cancer. Oncol Rep 2005;13:1153-8.

47. Tassi E, Gavazzi F, Albarello L, et al. Carcinoembryonic antigen-specific but not antiviral CD4+ T cell immunity is impaired in pancreatic carcinoma patients. J Immunol 2008;181:6595-603.

48. Ubukata H, Motohashi G, Tabuchi T, et al. Evaluations of interferon- $\gamma /$ interleukin-4 ratio and neutrophil/ lymphocyte ratio as prognostic indicators in gastric cancer patients. J Surg Oncol 2010;102:742-7.

49. Fridman WH, Pagès F, Sautès-Fridman C, et al. The immune contexture in human tumours: impact on clinical outcome. Nat Rev Cancer 2012;12:298-306.

50. Kryczek I, Banerjee M, Cheng P, et al. Phenotype, distribution, generation, and functional and clinical relevance of Th17 cells in the human tumor environments. Blood 2009;114:1141-9.

51. Lv L, Pan K, Li XD, et al. The accumulation and prognosis value of tumor infiltrating IL-17 producing cells in esophageal squamous cell carcinoma. PLoS One 2011;6:e18219.

52. Chen JG, Xia JC, Liang XT, et al. Intratumoral expression of IL-17 and its prognostic role in gastric adenocarcinoma patients. Int J Biol Sci 2011;7:53-60.

(English Language Editor: B. Meiser)
Cite this article as: Wei Z, Duan X, Li Q, Li Q, Wang Y. High expression of Ran binding protein 1 predicts poor outcomes in hepatocellular carcinoma patients: a Cancer Genome Atlas database analysis. J Gastrointest Oncol 2021;12(6):2966-2984. doi: 10.21037/jgo-21-541 
Supplementary

\begin{tabular}{|c|c|c|c|c|c|c|}
\hline Dataset & P-value & Type & Nums & Mean & STD & IQR \\
\hline \multirow{2}{*}{ HCCDB1 } & \multirow{2}{*}{0.0000139} & $\mathrm{HCC}$ & 100 & 8.411 & 0.4455 & 0.4509 \\
\hline & & Adjacent & 97 & 8.17 & 0.2993 & 0.4155 \\
\hline \multirow{2}{*}{ HCCDB4 } & \multirow{2}{*}{$3.40 \mathrm{E}-34$} & HCC & 240 & 8.774 & 0.5151 & 0.6367 \\
\hline & & Adjacent & 193 & 8.253 & 0.2734 & 0.3897 \\
\hline \multirow{2}{*}{ HCCDB6 } & \multirow{2}{*}{0.7408} & $\mathrm{HCC}$ & 225 & 3.938 & 0.2897 & 0.27 \\
\hline & & Adjacent & 220 & 3.948 & 0.3188 & 0.2712 \\
\hline \multirow{2}{*}{ HCCDB11 } & \multirow{2}{*}{0.03127} & HCC & 88 & 8.145 & 0.517 & 0.5862 \\
\hline & & Adjacent & 48 & 8.382 & 0.6427 & 0.8765 \\
\hline \multirow{2}{*}{ HCCDB12 } & \multirow{2}{*}{0.0000684} & $\mathrm{HCC}$ & 81 & 12.55 & 0.485 & 0.561 \\
\hline & & Adjacent & 80 & 12.28 & 0.3293 & 0.4637 \\
\hline \multirow{2}{*}{ HCCDB13 } & \multirow{2}{*}{0.8341} & $\mathrm{HCC}$ & 228 & 7.387 & 0.71 & 0.816 \\
\hline & & Adjacent & 168 & 7.374 & 0.5353 & 0.482 \\
\hline \multirow{2}{*}{ HCCDB15 } & \multirow{2}{*}{0.02363} & $\mathrm{HCC}$ & 351 & 10.07 & 0.6165 & 0.745 \\
\hline & & Adjacent & 49 & 9.959 & 0.2662 & 0.38 \\
\hline \multirow{2}{*}{ HCCDB16 } & \multirow{2}{*}{0.0004337} & $\mathrm{HCC}$ & 60 & 7.862 & 0.4071 & 0.471 \\
\hline & & Adjacent & 60 & 7.644 & 0.2173 & 0.2929 \\
\hline \multirow{2}{*}{ HCCDB17 } & \multirow{2}{*}{0.0000915} & HCC & 115 & 10.08 & 0.559 & 0.6525 \\
\hline & & Adjacent & 52 & 9.789 & 0.3682 & 0.4213 \\
\hline \multirow{2}{*}{ HCCDB18 } & \multirow{2}{*}{ 5.59E-23 } & $\mathrm{HCC}$ & 212 & 5.512 & 0.6093 & 0.7225 \\
\hline & & Adjacent & 177 & 5 & 0.311 & 0.38 \\
\hline
\end{tabular}

$\mathrm{B}$

\begin{tabular}{lll}
\hline Tissues & Staining & HPA065931 \\
\hline \multirow{3}{*}{ HCC } & High & 2 \\
& Medium & 2 \\
& Low & 2 \\
Liver & Not detected & 6 \\
\hline
\end{tabular}

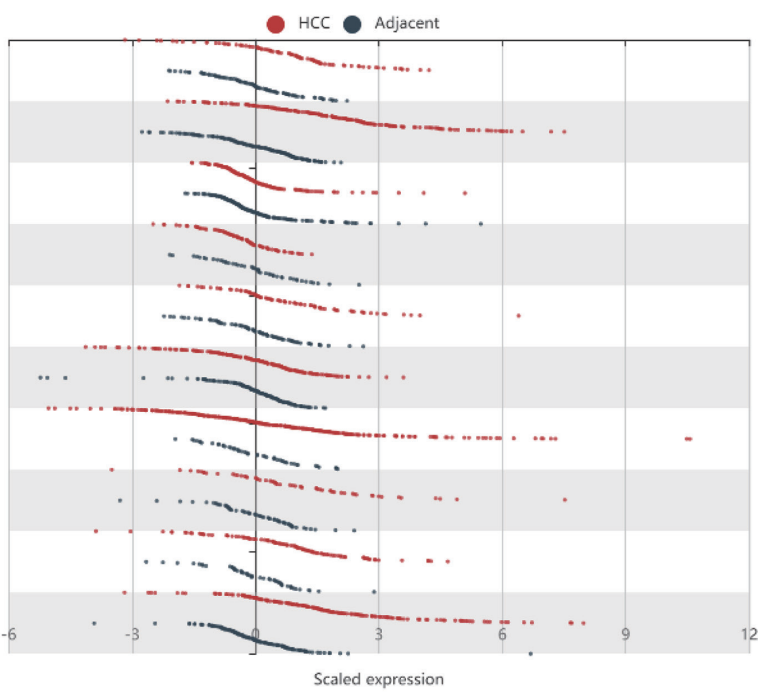

C

Antibody HPA065931

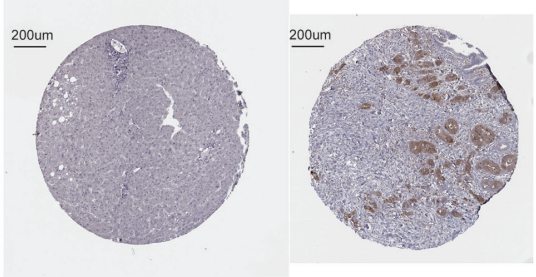

Liver

HCC

Figure S1 The expression level of RANBP1 in HCC. (A) Chart and plot showing the expression of RANBP1 in tumor tissues and the adjacent normal tissues, according to $t$-test using the Integrative Molecular Database for Hepatocellular Carcinoma (HCCDB). (B) The protein expression of RANBP1 in HCC from the HPA. (C) Distribution of RANBP1 in HCC. The staining method was immunohistochemistry staining $(200 \mu \mathrm{m})$. Color images are available online (Open access and available on the Human Protein Atlas: https:// images.proteinatlas.org/65931/162102_A_8_4_medium.jpg; https://images.proteinatlas.org/65931/162101_B_9_3.jpg). RANBP1, Ranspecific binding protein 1; HCC, hepatocellular carcinoma; HPA, Human Protein Atlas. 
Table S1 Univariate and multivariate Cox proportional hazards analysis of RANBP1 expression and progress-free interval for patients with HCC in The Cancer Genome Atlas cohort

\begin{tabular}{|c|c|c|c|c|}
\hline Characteristics & \multicolumn{2}{|c|}{ Univariate analysis } & \multicolumn{2}{|c|}{ Multivariate analysis } \\
\hline T stage (T2 \& T3 \& T4 vs. T1) & $2.384(1.757-3.235)$ & $<0.001$ & $0.700(0.092-5.335)$ & 0.730 \\
\hline N stage (N1 vs. N0) & $1.385(0.342-5.611)$ & 0.648 & & \\
\hline M stage (M1 vs. M0) & $3.442(1.080-10.967)$ & 0.037 & $2.272(0.671-7.692)$ & 0.187 \\
\hline Histologic grade (G3\&G4 vs. G1\&G2) & $1.154(0.852-1.564)$ & 0.354 & & \\
\hline Residual tumor (R1\&R2 vs. R0) & $1.515(0.841-2.730)$ & 0.167 & & \\
\hline Age (>60 vs. $\leq 60)$ & $0.952(0.710-1.275)$ & 0.740 & & \\
\hline Fibrosis Ishak score $(1 / 2 \& 3 / 4 \& 5 / 6$ vs. 0$)$ & $1.369(0.910-2.061)$ & 0.132 & & \\
\hline $\begin{array}{l}\text { Adjacent hepatic tissue inflammation (mild \& severe } \\
\text { vs. none) }\end{array}$ & $1.238(0.863-1.776)$ & 0.246 & & \\
\hline Vascular invasion (yes vs. no) & $1.682(1.196-2.364)$ & 0.003 & $1.631(1.000-2.658)$ & 0.050 \\
\hline RANBP1 (high vs. low) & $1.578(1.175-2.118)$ & 0.002 & $1.805(1.187-2.744)$ & 0.006 \\
\hline
\end{tabular}

$\mathrm{Cl}$, confidence interval; HR, hazard ratio; RANBP1, Ran-specific binding protein 1. 
Table S2 Univariate and multivariate Cox proportional hazards analysis of RANBP1 expression and DSS for patients with HCC in TCGA cohort

\begin{tabular}{|c|c|c|c|c|}
\hline Characteristics & \multicolumn{2}{|c|}{ Univariate analysis } & \multicolumn{2}{|c|}{ Multivariate analysis } \\
\hline T stage (T2\&T3\&T4 vs. T1) & $2.807(1.733-4.546)$ & $<0.001$ & $1.857(0.757-4.557)$ & 0.176 \\
\hline $\mathrm{N}$ stage (N1 vs. N0) & $3.562(0.858-14.785)$ & 0.080 & $1.010(0.098-10.360)$ & 0.993 \\
\hline M stage (M1 vs. M0) & $5.102(1.230-21.161)$ & 0.025 & $4.563(0.858-24.277)$ & 0.075 \\
\hline Histologic grade (G3 \& G4 vs. G1 \& G2) & $1.119(0.703-1.780)$ & 0.636 & & \\
\hline Residual tumor (R1 \& R2 vs. R0) & $1.640(0.711-3.782)$ & 0.246 & & \\
\hline Age $(>60$ vs. $\leq 60)$ & $0.880(0.566-1.370)$ & 0.573 & & \\
\hline Fibrosis Ishak score $(1 / 2 \& 3 / 4 \& 5 / 6$ vs. 0$)$ & $0.921(0.478-1.774)$ & 0.806 & & \\
\hline $\begin{array}{l}\text { Adjacent hepatic tissue inflammation (mild \& severe } \\
\text { vs. none) }\end{array}$ & $1.446(0.791-2.645)$ & 0.231 & & \\
\hline Vascular invasion (yes vs. no) & $1.284(0.710-2.319)$ & 0.408 & & \\
\hline RANBP1 (high vs. low) & $2.123(1.341-3.360)$ & 0.001 & $2.852(1.178-6.903)$ & 0.020 \\
\hline
\end{tabular}

$\mathrm{Cl}$, confidence interval; HR, hazard ratio. 